\title{
Scenario-based risk evaluation
}

\author{
Ruodu Wang ${ }^{1} \cdot$ Johanna F. Ziegel ${ }^{2}$
}

Received: 6 March 2020 / Accepted: 30 April 2021 / Published online: 3 September 2021

(c) The Author(s) 2021

\begin{abstract}
Risk measures such as expected shortfall (ES) and value-at-risk (VaR) have been prominent in banking regulation and financial risk management. Motivated by practical considerations in the assessment and management of risks, including tractability, scenario relevance and robustness, we consider theoretical properties of scenariobased risk evaluation. We establish axiomatic characterisations of scenario-based risk measures that are comonotonic-additive or coherent, and we obtain a novel ES-based representation result. We propose several novel scenario-based risk measures, including various versions of Max-ES and Max-VaR, and study their properties. The theory is illustrated with financial data examples.
\end{abstract}

Keywords Scenarios - Risk measures - Basel Accords · Stress adjustment · Dependence adjustment

Mathematics Subject Classification (2020) 91G70 • 91B05

JEL Classification C69 - G28

\section{Introduction}

\subsection{Background}

Risk measures are used in various contexts in banking and insurance, such as regulatory capital calculation, optimisation, decision making, performance analysis, and risk pricing. In practice, risk measures have to be estimated from data. Therefore, it is often argued that one has to use a law-based risk measure (or a statistical functional),

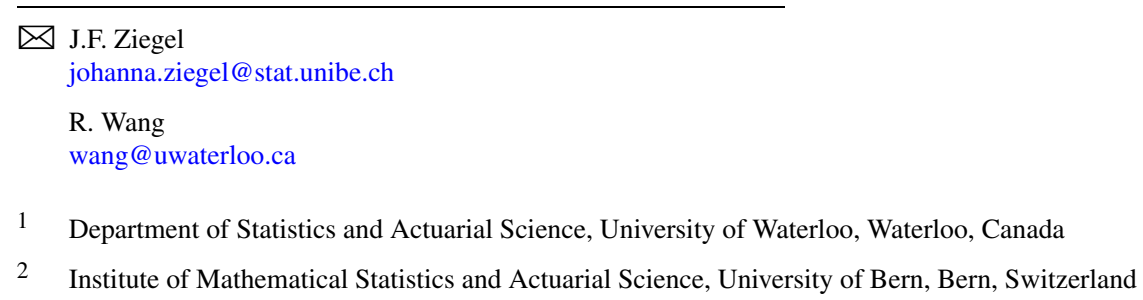


such as a value-at-risk (VaR) or an expected shortfall (ES), which are both standard risk measures used in banking and insurance.

However, even assuming that the distribution of a risk is accurately obtained, it need not be able to comprehensively describe the nature of the risk. From the regulatory perspective, a regulator is more concerned about the behaviour of a risk in an adverse environment, e.g. during a catastrophic financial event; see e.g. Acharya et al. [1] for related discussions. Only the distribution of the risk may be not enough to distinguish a potentially huge loss in a financial crisis from a potentially huge loss in a common economy but no loss in a financial crisis. As another simple example, the profit/loss from a lottery and that from an insurance contract may have the same distribution, but they represent very different types of risks and can have very different effects on the decision maker or the society. Therefore, it may be useful to evaluate a risk under different stress scenarios. Summing up these evaluations in a single number would necessarily lead to a non-law-based risk measure.

Finally, it is usually unrealistic to assume that the distribution of a risk may be accurately obtained. Model uncertainty is a central component of the current challenges in risk measurement and regulation, and its importance in practice has been pivotal after the 2007 financial crisis (see e.g. [36]) in both the banking (e.g. [5]) and insurance sectors (e.g. [26]). Model uncertainty may be due to statistical/parameter uncertainty or, more generally, structural uncertainty of the model or of the economic system. A robust approach should take into account the distribution of the underlying risk under several plausible model assumptions.

In the framework of Basel III \& IV [5], the standard risk measure for market risk is an expected shortfall $\left(\mathrm{ES}_{p}\right)$ at level $p=0.975$. Thus, the Basel Committee on Banking Supervision has opted for a law-based risk measure. However, while ES is the basic building block for market risk assessment, the initial ES estimates are subsequently modified. In particular, two important adjustments are a stress adjustment and a dependence adjustment [5, pp. 52-69], which then leads to the capital charge for modellable risk factors (abbreviated as IMCC in [5]).

The aim of this paper is to present a theoretical approach to the construction of risk measures that incorporates modifications such as a stress and dependence adjustment of an initial law-based risk measure into the risk measure itself. We call such risk measures scenario-based risk measures; see Definition 2.2. Our approach has the advantage that the final result of the risk estimation can be understood theoretically, and properties such as coherence and comonotonic additivity can be studied not only for the initial law-invariant risk measure, but for the final risk measure that is the relevant output for further actions and decisions, such as the IMCC in the Basel III \& IV framework.

Before presenting our theoretical framework, let us give some details on the latest regulatory framework of the Basel Committee on Banking Supervision to illustrate how they deal with the issues mentioned above.

In the Fundamental Review of the Trading Book (FRTB) of Basel III \& IV [5] for market risk, the time horizon is 10 days (two trading weeks), and each risk position (random loss) is modelled as a function of risk factors, such as equity prices, interest rates, credit spreads and volatilities. Each risk factor is adjusted according to their category of liquidity (see Li and Xing [32] for a precise mathematical formulation). For simplicity, we consider a linear portfolio in the illustration below. Let 
$X=\sum_{i=1}^{n} X_{i}$ be the aggregate portfolio loss on a given day, where $X_{1}, \ldots, X_{n}$ are the corresponding risk factors in the aggregation (with weights included). Below we outline two adjustments that the FRTB uses to calculate the regulatory capital.

(i) Stress adjustment

(a) Specify a set $R$ of reduced risk factors which has a sufficiently long history of observation (at least spanning back to and including 2007), such that the ratio

$$
\theta=\max \left\{\frac{\operatorname{ES}_{F}(X)}{\operatorname{ES}_{R}(X)}, 1\right\}
$$

is less than 4/3, where $\operatorname{ES}_{F}(X)=\operatorname{ES}_{p}\left(\sum_{i=1}^{n} X_{i}\right)$ is the current ES value calculated using all risk factors, and $\mathrm{ES}_{R}(X)=\mathrm{ES}_{p}\left(\sum_{i \in R} X_{i}\right)$ is the current ES value calculated using the reduced risk factors. The ratio $\theta$ is treated like a constant and only needs to be updated weekly.

(b) Compute ES for a model with the reduced risk factors, "calibrated to the most severe 12-month period of stress", and denote this by $\operatorname{ES}_{R, S}(X)$. The period of "most severe stress", also called the stress scenario, corresponds to the rolling window of data of length one year that leads to the maximum possible value of ES using the reduced risk factor model [6, p. 6]. Mathematically, $\mathrm{ES}_{R, S}(X)$ involves taking a maximum over a set $\mathcal{Q}$ of distributions estimated from sequences of data of length one year (many of them overlapping), namely

$$
\mathrm{ES}_{R, S}(X)=\max _{Q \in \mathcal{Q}} \mathrm{ES}_{p}^{Q}\left(\sum_{i \in R} X_{i}\right)
$$

(c) Use the formula

$$
\widetilde{\mathrm{ES}}(X)=\mathrm{ES}_{R, S}(X) \times \theta
$$

to get the stress-adjusted ES value.

In particular, if the portfolio loss is modelled by only risk factors of sufficiently long history (spanning back to 2007), then $R=\{1, \ldots, n\}$ and the adjusted ES value is

$$
\widetilde{\mathrm{ES}}(X)=\max _{Q \in \mathcal{Q}} \operatorname{ES}_{p}^{Q}\left(\sum_{i=1}^{n} X_{i}\right)=\max _{Q \in \mathcal{Q}} \operatorname{ES}_{p}^{Q}(X)
$$

(ii) Dependence adjustment

(a) Risk factors in the portfolio are grouped into a range of broad regulatory risk classes (interest rate risk, equity risk, foreign exchange risk, commodity risk and credit spread risk). For the stress scenario (see (i)(b)), compute the ES of each risk class (according to (i)), and denote their sum by $\widetilde{\mathrm{ES}}_{C}(X)$. By comonotonic additivity and subadditivity of ES (see Sect. 2 for details), this calculation is equivalent to using a model where all classes of risk factors are comonotonic ("non-diversified"), and 
it represents the worst-case value of ES among all possible dependence structures (e.g. Embrechts et al. [18]).

(b) Use the formula

$$
\mathrm{ES}(X)=\lambda \widetilde{\mathrm{ES}}(X)+(1-\lambda) \widetilde{\mathrm{ES}}_{C}(X)
$$

where $\lambda$ is a constant (right now, $\lambda$ is chosen as 0.5 ). The quantity $\operatorname{ES}(X)$ is called the IMCC of the portfolio.

Intuitively, the logic behind adjustment (i) is that risk assessment should be made based on stressed financial periods, and that behind adjustment (ii) is that the dependence structure between risk factors is difficult to specify and a worst-case value is combined with the original model to protect from overly optimistic diversification effects in the model specification. In addition to (i) and (ii), the IMCC value will finally be adjusted by using the maximum of its present calculation and a moving average calculation of 60 days times a constant (currently 1.5). See Embrechts et al. [18, 19] for discussions on the aggregation of risk measures under dependence uncertainty.

In summary, in the FRTB, the ES of the same portfolio is estimated under different scenarios and models: stress (stressed, non-stressed), and dependence (diversified, non-diversified). These values are aggregated with mainly two operations (iteratively): maximum and linear combination. In Theorem 3.8, we show that these two operations indeed are the two most crucial operations which lead to coherence in the sense of Artzner et al. [4] for scenario-based risk measures. Section 5.2 contains a detailed data analysis for the stress adjustment (i) outlined above.

We briefly mention two other prominent examples of risk evaluation using scenarios. First, the margin requirements calculation developed by the Chicago Mercantile Exchange [9] relies on the maximum of the portfolio loss over several specified hypothetical scenarios [33, p. 63]. Our data example in Sect. 5.1 is similar to this approach. The second example comes from the practice of credit rating, where a structured finance security (e.g. a defaultable bond) is rated according to its behaviour (conditional distributions) under each economic stress scenario. This approach, in different specific forms, appears in Guo et al. [24, Sect. 5.2].

In this paper, we propose an axiomatic framework of scenario-based risk evaluation which has the three merits mentioned above and is consistent with many existing risk measurement procedures including the above examples. We shall keep the Basel formulas as our primary example in mind.

\subsection{Our contribution and the structure of the paper}

In Sect. 2, we introduce scenario-based risk measures. They include classic lawbased risk measures, non-law-based risk measures such as the systemic risk measures CoVaR and CoES (Adrian and Brunnermeier [2]), and many practically used risk calculation principles such as the Basel formulas for market risk, the margin requirements by the Chicago Mercantile Exchange, and the common rating measures used in credit rating, as mentioned above. Axiomatic characterisations of scenariobased risk measures are studied in Sect. 3. In particular, we characterise scenariobased comonotonic-additive as well as coherent risk measures, where many surprising mathematical challenges emerge. In Sect. 4, we introduce Max-ES and Max-VaR 
and related families of risk measures and study their properties. Data analyses are given in Sect. 5, highlighting the broad range of possible interpretations of scenarios. In particular, scenario-based risk measures can be easily implemented for stress analysis and capital calculation.

Our framework builds upon the axiomatic theory of coherent risk measures as pioneered by Artzner et al. [4]. A comprehensive review on risk measures can be found in the book of Föllmer and Schied [22, Chap. 4].

Distinguished from the literature, the main mathematical challenges in our paper come from the novel framework of treating risk measures as functionals on a space of random variables (as in the traditional setting) through the distributions of a random variable under each scenario (probability measure). The mathematical structure is very different from that with tuples of distributions as inputs, such as the classic framework of Anscombe and Aumann [3] (see also Gilboa and Schmeidler [23], Cerreia-Vioglio et al. [8], Hansen and Marinacci [25]) in decision theory under ambiguity. The distributions of a random variable under each scenario are not arbitrary. Shen et al. [41] recently studied the issue of compatibility between distributions and scenarios; for instance, if $Q_{1}$ and $Q_{2}$ are mutually equivalent and the distribution of $X$ is uniform under $Q_{1}$, then it cannot be normal under $Q_{2}$. If tuples of distributions are used as inputs, the "geometry" (interdependence) of the set of scenarios does not play a role in the characterisation results. This is in sharp contrast to our framework, where the interdependence among the set of scenarios plays an important role in the characterisation of risk measures. See the detailed discussion in Sect. 3.1 and Example 3.1, as well as Theorem 3.8, where the choice of the set of scenarios clearly matters for the characterisation result.

Kou et al. [29] and Kou and Peng [28] studied properties of risk measures based on scenarios from different angles than ours. Various scenario-based risk measures also appear in Zhu and Fukushima [44], Zymler et al. [45], Adrian and Brunnermeier [2], Righi [37] in different disguises. Our contribution is to study the consequence of the scenario-based property instead of specific examples; thus our results yield axiomatic support for specific risk measures in the above literature. For recent developments of risk measures, including various practical issues of statistical analysis, robustness, model uncertainty and optimisation, we refer to Fissler and Ziegel [20], Cambou and Filipović [7], Krätschmer et al. [30], Du and Escanciano [15], Embrechts et al. [17], Wang and Zitikis [42] and the references therein.

\section{Scenario-based risk measures}

\subsection{Definitions}

Let $(\Omega, \mathcal{F})$ be a measurable space and $\mathcal{P}$ the set of all probability measures on $(\Omega, \mathcal{F})$. For any $Q \in \mathcal{P}$, write $F_{X, Q}$ for the cumulative distribution function (cdf) of a random variable $X$ under $Q$, i.e., $F_{X, Q}(x)=Q[X \leq x]$ for $x \in \mathbb{R}$, and write $X \sim_{Q} F$ if $F=F_{X, Q}$. For two random variables $X$ and $Y$ and a probability measure $Q$, we write $X \stackrel{\mathrm{d}}{=} Q Y$ if $F_{X, Q}=F_{Y, Q}$. For any cdf $F$, its generalised inverse is defined as $F^{-1}(t)=\inf \{x \in \mathbb{R}: F(x) \geq t\}$ for $t \in(0,1]$. Let $\mathcal{X}$ be the space of bounded 
random variables on $(\Omega, \mathcal{F})$ and $\mathcal{Y}$ a convex cone of random variables containing $\mathcal{X}$, representing the set of random variables of interest, which are possibly unbounded. We fix $\mathcal{X}$ throughout, whereas $\mathcal{Y}$ is specific to the functional considered. For instance, when considering the expectation $\mathbb{E}^{Q}$ for some $Q \in \mathcal{P}$, its domain $\mathcal{Y}$ is often chosen as the space of $Q$-integrable random variables, which depends on the choice of $Q$. However, it does not hurt to think of $\mathcal{Y}=\mathcal{X}$ for the main part of the paper. A probability measure $\mathbb{P} \in \mathcal{P}$ is chosen as a reference probability measure in this paper, and it may be interpreted as the real-world probability measure in some applications.

In this paper, we use the term scenario for a probability measure $Q \in \mathcal{P}$. The reason behind this choice of terminology is from the perspective of scenario analysis, as in the following example. This example will be referred to a few times throughout the paper.

Example 2.1 Let $\Theta$ be a random economic factor taking values in a set $K$ and $Q_{\theta}[\cdot]=\mathbb{P}[\cdot \mid \Theta=\theta], \theta \in K$, regular conditional probabilities with reference to $\Theta$. The set $\{\Theta=\theta\} \in \mathcal{F}$ represents a possible economic event for each $\theta \in K$. To analyse the behaviour of a risk $X$ under each scenario $\{\Theta=\theta\}, \theta \in K$, the respective distributions of $X$ under the probability measures $Q_{\theta}$ are of interest.

Suppose that there is a collection $\mathcal{Q}$ of scenarios of interest. As mentioned in the introduction, there may be different interpretations for the set $\mathcal{Q}$. In what follows, we take a collection of scenarios of interest and do not distinguish among the interpretations. If a risk (random loss) $X$ and another risk $Y$ have the same distribution under all relevant scenarios in $\mathcal{Q}$, they should be assigned identical riskiness, whatever sense of riskiness we speak of. This leads to the following definition.

Definition 2.2 For a family of scenarios $\emptyset \neq \mathcal{Q} \subseteq \mathcal{P}$, a mapping $\rho: \mathcal{Y} \rightarrow(-\infty, \infty]$ is $\mathcal{Q}$-based if $\rho(X)=\rho(Y)$ for $X, Y \in \mathcal{Y}$ whenever $X \stackrel{\text { d }}{=}{ }_{Q} Y$ for all $Q \in \mathcal{Q}$.

To put the above concept into risk management, we focus on $\mathcal{Q}$-based risk measures. A risk measure is a mapping from $\mathcal{Y}$ to $(-\infty, \infty$ ] with $\rho(X)<\infty$ for a bounded $X$. We use the term risk measure in a broad sense, as it also includes deviation measures (such as the variance) and other risk functionals. To keep things concise, our main examples are traditional risk measures such as VaR and ES, although our framework includes deviation measures. For the latter, see Rockafellar et al. [38]. In this paper, we adopt the sign convention as in McNeil et al. [33, Chap. 2]: for a risk $X \in \mathcal{Y}$, losses are represented by positive and profits by negative values of $X$.

An immediate example of a $\mathcal{Q}$-based risk measure is one that depends on the joint law of a risk and an economic factor $\Theta$ as in Example 2.1. By choosing the set $\mathcal{Q}=\{\mathbb{P}[\cdot \mid \Theta=\theta]: \theta \in K\}$, we see that $\rho$ is $\mathcal{Q}$-based if and only if $\rho(X)$ is determined by the joint distribution of $(X, \Theta)$. This setting includes the systemic risk measures CoVaR and CoES, which are evaluated based on conditional distributions of risks given events (see Adrian and Brunnermeier [2]). For a fixed random variable $S$ (the system) and $p \in(0,1)$, the systemic risk measure CoVaR of the institution loss $X \in \mathcal{Y}$ is defined as

$$
\operatorname{CoVaR}_{p}^{S}(X)=\operatorname{VaR}_{p}^{\mathbb{P}}\left(S \mid X=\operatorname{VaR}_{p}^{\mathbb{P}}(X)\right),
$$


and the other systemic risk measure CoES is defined as

$$
\operatorname{CoES}_{p}^{S}(X)=\mathbb{E}^{\mathbb{P}}\left[S \mid S \geq \operatorname{CoVaR}_{p}^{S}(X)\right], \quad X \in \mathcal{Y} .
$$

Since CoVaR and CoES are determined by the joint distribution of $(X, S)$, they are $\mathcal{Q}$-based risk measures for $\mathcal{Q}=\{\mathbb{P}[\cdot \mid S=s]: s \in \mathbb{R}\}$.

Clearly, $\mathcal{Q}$-based risk measures are generalisations of law-based (single-scenariobased) risk measures, which are determined by the law of a random variable on a given probability space. Thus $\mathcal{Q}$-based risk measures bridge law-based ones and generic ones, by noting the relationship (assuming $\mathbb{P} \in \mathcal{Q}$ )

$$
\underbrace{\{\mathbb{P}\}}_{\text {law-based }} \subseteq \underbrace{\mathcal{Q}}_{\mathcal{Q} \text {-based }} \subseteq \underbrace{\mathcal{P}}_{\text {generic }} .
$$

Some immediate facts about $\mathcal{Q}$-based risk measures are summarised in the following:

(i) All risk measures on $\mathcal{Y}$ are $\mathcal{P}$-based. In fact, if $X \stackrel{\mathrm{d}}{=}{ }_{Q} Y$ for all $Q \in \mathcal{P}$, then $X=Y$ (equality as mappings).

(ii) If $\mathcal{Q}_{1} \subseteq \mathcal{Q}_{2} \subseteq \mathcal{P}$, then a $\mathcal{Q}_{1}$-based risk measure is also $\mathcal{Q}_{2}$-based.

(iii) For $\mathcal{Q}_{1}, \ldots, \mathcal{Q}_{n} \subseteq \mathcal{P}$, let $\rho_{i}: \mathcal{Y} \rightarrow \mathbb{R}$ be $\mathcal{Q}_{i}$-based, $i=1, \ldots, n$, and $\mathcal{Q}=\bigcup_{i=1}^{n} \mathcal{Q}_{i}$. For any $f: \mathbb{R}^{n} \rightarrow \mathbb{R}$, the mapping $f \circ\left(\rho_{1}, \ldots, \rho_{n}\right): \mathcal{Y} \rightarrow \mathbb{R}$ is then $\mathcal{Q}$-based.

To see that claim (i) holds, let $\omega \in \Omega$ and choose as $Q$ the Dirac measure in $\omega$, i.e., $Q: \mathcal{F} \rightarrow \mathbb{R}, A \mapsto \mathbb{1}_{A}(\omega)$. The distributions of $X$ and $Y$ under $Q$ are simply the point masses at $X(\omega)$ and $Y(\omega)$, respectively. Therefore, $X \stackrel{\mathrm{d}}{=} Q Y$ implies that $X(\omega)=Y(\omega)$.

Next we introduce a special type of collections of probability measures which fits naturally into the context of Example 2.1.

Definition 2.3 A collection of probability measures $\mathcal{Q} \subseteq \mathcal{P}$ is mutually singular if there exist mutually disjoint sets $A_{Q} \in \mathcal{F}, Q \in \mathcal{Q}$, such that $Q\left[A_{Q}\right]=1$ for $Q \in \mathcal{Q}$. We say that a tuple $\left(Q_{1}, \ldots, Q_{n}\right) \in \mathcal{P}^{n}$ is mutually singular if $\left\{Q_{1}, \ldots, Q_{n}\right\}$ is mutually singular and any two of $Q_{1}, \ldots, Q_{n}$ are non-identical.

An example of this type would be to take $Q_{i}[B]=\mathbb{P}\left[B \mid A_{i}\right]$ for $B \in \mathcal{F}$, where $A_{1}, \ldots, A_{n}$ is a partition of $\Omega$ with $\mathbb{P}\left[A_{i}\right]>0$ for $i=1, \ldots, n$. That is, each $Q_{i}$ amplifies the probability of the events $A_{i}$ of interest, commonly seen e.g. in importance sampling. In Example 2.1, $\mathcal{Q}=\left\{Q_{\theta}: \theta \in K\right\}$ is mutually singular.

Remark 2.4 In this paper, scenarios are treated in a generic sense. They may have different interpretations in different contexts. In a statistical context, they may represent different values of an estimated parameter in the model of the risk. In a simulationbased model, they may represent different parameters in the simulation dynamics, or different probabilities used in importance sampling. In a regulatory framework, they may represent different economic situations that the regulator is concerned about. In a financial market, to assess a contingent payoff, one may need to incorporate its distribution under the pricing measure and under the physical measure, under multiple 
pricing measures or with different heterogeneous opinions about the physical probability measure; these situations naturally require a risk measure determined by the distribution of the risk under different measures.

\subsection{Preliminaries on risk measures}

We adopt the terminology introduced in Föllmer and Schied [21]. A risk measure $\rho: \mathcal{Y} \rightarrow(-\infty, \infty]$ is called cash-invariant if $\rho(X+c)=\rho(X)+c$ for $c \in \mathbb{R}$ and $X \in \mathcal{Y}$, monotone if $\rho(X) \leq \rho(Y)$ for $X, Y \in \mathcal{Y}$ with $X \leq Y$, positively homogeneous if $\rho(\lambda X)=\lambda \rho(X)$ for $\lambda \in(0, \infty)$ and $X \in \mathcal{Y}$, and subadditive if $\rho(X+Y) \leq \rho(X)+\rho(Y)$ for $X, Y \in \mathcal{Y}$. A risk measure is called monetary if it is monotone and cash-invariant, and coherent if it is monetary, positively homogeneous and subadditive. Two random variables $X$ and $Y$ on $(\Omega, \mathcal{F})$ are called comonotonic if $\left(X(\omega)-X\left(\omega^{\prime}\right)\right)\left(Y(\omega)-Y\left(\omega^{\prime}\right)\right) \geq 0$ for all $\omega, \omega^{\prime} \in \Omega$. A risk measure $\rho$ is comonotonic-additive if $\rho(X+Y)=\rho(X)+\rho(Y)$ whenever $X$ and $Y$ are comonotonic.

Let us recall some classic risk measures based on a single scenario $Q \in \mathcal{P}$. The most popular risk measures in banking and insurance regulation are value-at-risk $(\mathrm{VaR})$ and expected shortfall (ES), calculated under a fixed probability measure $Q \in \mathcal{P}$. We refer to them as $Q$-VaR and $Q$-ES, respectively. For these risk measures, their domain $\mathcal{Y}$ can be chosen as any convex cone of random variables containing $\mathcal{X}$, possibly the entire set of random variables. For $p \in(0,1], \operatorname{VaR}_{p}^{Q}: \mathcal{Y} \rightarrow(-\infty, \infty]$ is defined as

$$
\operatorname{VaR}_{p}^{Q}(X)=\inf \{x \in \mathbb{R}: Q[X \leq x] \geq p\}=F_{X, Q}^{-1}(p), \quad X \in \mathcal{Y},
$$

and for $p \in(0,1), \operatorname{ES}_{p}^{Q}: \mathcal{Y} \rightarrow(-\infty, \infty]$ is defined as

$$
\operatorname{ES}_{p}^{Q}(X)=\frac{1}{1-p} \int_{p}^{1} \operatorname{VaR}_{q}^{Q}(X) \mathrm{d} q, \quad X \in \mathcal{Y} .
$$

Since $-\infty<\operatorname{VaR}_{p}^{Q}(X) \leq \operatorname{VaR}_{q}^{Q}(X)<\infty$ for $p \leq q<1$, the integral (2.2) is well defined. In addition, we let $\operatorname{ES}_{1}^{Q}(X)=\operatorname{VaR}_{1}^{Q}(X)$.

For a specified scenario $Q, Q$-VaR and $Q$-ES belong to the class of distortion risk measures. Define the sets of functions

$$
\mathcal{G}=\{g:[0,1] \rightarrow[0,1]: g \text { is increasing with } g(0)=0 \text { and } g(1)=1\},
$$

and $\mathcal{G}_{\mathrm{cv}}=\{g \in \mathcal{G}: g$ is concave $\}$. In this paper, the terms "increasing", "decreasing" and "set inclusion" are in the non-strict sense. A $Q$-distortion risk measure is defined as

$$
\rho_{g}^{Q}(X)=\int_{-\infty}^{0}(g \circ Q[X>x]-1) \mathrm{d} x+\int_{0}^{\infty} g \circ Q[X>x] d x, \quad X \in \mathcal{X}_{g},
$$

where $g \in \mathcal{G}$ is called the distortion function of $\rho_{g}^{Q}$ and $\mathcal{X}_{g}$ is the set of random variables such that the first integral in (2.3) is finite. Then $\rho_{g}^{Q}: \mathcal{X}_{g} \rightarrow(-\infty, \infty]$ is a 
well-defined risk measure. The set $\mathcal{X}_{g}$ always contains $\mathcal{X}$. A $Q$-spectral risk measure is a $Q$-distortion risk measure with a concave distortion function. A $Q$-distortion risk measure is always monetary, positively homogeneous and comonotonic-additive. A $Q$-spectral risk measure is in addition coherent. $\operatorname{VaR}_{p}^{Q}$ has the distortion function $g(x)=\mathbb{1}_{\{x>1-p\}}, x \in[0,1]$, and $\operatorname{ES}_{p}^{Q}$ has the distortion function

$$
g(x)=(1 /(1-p)) \min \{x, 1-p\}, \quad x \in[0,1] .
$$

For the above properties of distortion risk measures, see Föllmer and Schied [22, Sect. 4.7].

\section{Axiomatic characterisations}

In this section, we establish axiomatic characterisations of $\mathcal{Q}$-based comonotonicadditive risk measures as well as $\mathcal{Q}$-based coherent risk measures. We focus on a finite collection $\mathcal{Q}$ and the set of bounded random variables, that is, $\mathcal{Y}=\mathcal{X}$. The latter is reasonable when we look for axiomatic characterisation results since properties on $\mathcal{Y} \supseteq \mathcal{X}$ imply those on $\mathcal{X}$.

Throughout this section, $n$ is a positive integer, and $Q=\left(Q_{1}, \ldots, Q_{n}\right)$ is a vector of measures, where $Q_{1}, \ldots, Q_{n} \in \mathcal{P}$ are (pre-assigned) probability measures on $(\Omega, \mathcal{F})$, and $\mathcal{Q}=\left\{Q_{1}, \ldots, Q_{n}\right\}$ is the set of these measures. The dimensionality of $Q$ and the cardinality of $\mathcal{Q}$ only differ if some of $Q_{1}, \ldots, Q_{n}$ are identical. If $Q_{1}, \ldots, Q_{n}$ are distinct, the mutual singularity of $Q$ is equivalent to that of $\mathcal{Q}$. Write $\mathbf{0}=(0, \ldots, 0) \in \mathbb{R}^{n}$ and $\mathbf{1}=(1, \ldots, 1) \in \mathbb{R}^{n}$. We say that $P \in \mathcal{P}$ dominates $\mathcal{Q}$ if $Q \ll P$ for all $Q \in \mathcal{Q}$, that is, if $Q$ is absolutely continuous with respect to $P$ for all $Q \in \mathcal{Q}$. We say that $Q$ (or $\mathcal{Q}$ ) is atomless if $\left(\Omega, \mathcal{F}, Q_{i}\right)$ is atomless for each $i=1, \ldots, n$. Recall that a probability space $(\Omega, \mathcal{F}, Q)$ is atomless if and only if there exists a uniform random variable $U$ on $(\Omega, \mathcal{F}, Q)$.

\subsection{Novelty and challenges of our framework}

We first illustrate the distinction of our framework to other results in the literature, as this is mathematically quite subtle. The main message is that the interdependence among $Q_{1}, \ldots, Q_{n}$ (e.g. whether they are mutually singular or not) matters for the risk measure axioms in our framework, whereas this is irrelevant for results in the literature on scenario-based functionals (e.g. Cerreia-Vioglio et al. [8], Kou and Peng [28]).

The following simple example illustrates an interesting feature of scenario-based risk measures which is in sharp contrast to classic law-based risk measures.

Example 3.1 For $P, Q \in \mathcal{P}$, we define the $\{P, Q\}$-based risk measure $\rho$ as

$$
\rho(X)=2 \mathbb{E}^{P}[X]-\mathbb{E}^{Q}[X], \quad X \in \mathcal{X} .
$$

Note that $2 P-Q \in \mathcal{P}$ if and only if $2 P \geq Q$, and under this condition, $\rho$ is the expectation under the probability measure $2 P-Q$. If $2 P \geq Q$ fails to hold, then $\rho$ is not monotone. Hence $\rho$ is coherent if and only if $2 P \geq Q$. 
To understand the implications of Example 3.1, we look at the notion of the usual stochastic order. For $Q \in \mathcal{P}$ and two random variables $X, Y$, we write $X \precsim_{\text {st }}^{Q} Y$ if $F_{X, Q}(x) \geq F_{Y, Q}(x)$ for all $x \in \mathbb{R}$. We say that $\rho$ is $\mathcal{Q}$-monotone if $\rho(X) \leq \rho(Y)$ for

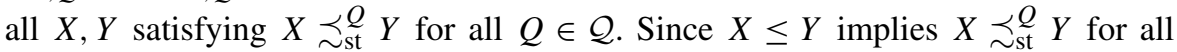
$Q \in \mathcal{Q}$, a risk measure $\rho$ is monotone if it is $\mathcal{Q}$-monotone. As a well-known property, in the case of $\mathcal{Q}=\{P\}$ being a singleton, a $\{P\}$-based risk measure is monotone if and only if it is $\{P\}$-monotone. However, the risk measure $\rho$ in Example 3.1 is in general not $\mathcal{Q}$-monotone (see Proposition 3.6), but it is monotone and coherent if $2 P \geq Q$. This is in sharp contrast to the case of $\{P\}$-based risk measures.

The above observation suggests that the relationship among $P$ and $Q$ matters for the properties of $\rho$. To determine whether $\rho$ is a coherent risk measure, we need to specify two things: first, how $\rho$ incorporates the distributions of the risk under each scenario (i.e., the mapping $\left.\left(F_{X, Q}\right)_{Q \in \mathcal{Q}} \mapsto \rho(X)\right)$; second, how these scenarios interact with each other. In the case of $\{P\}$-based risk measures, the mapping $F_{X, P} \mapsto \rho(X)$ solely determines properties of the risk measure, whereas the choice of the measure $P$ is irrelevant. For instance, $\operatorname{ES}_{p}^{P}$ and $\mathbb{E}^{P}$ are always coherent risk measures regardless of the choice of $P$.

The above discussion is related to the popular notion of consequentialism in decision theory in the framework of Anscombe and Aumann [3]. In the framework of consequentialism, two random outcomes $X$ and $Y$ (called Anscombe-Aumman acts) are compared via a preference model which aggregates the tuples of distributions $\left(F_{X, Q}\right)_{Q \in \mathcal{Q}}$ and $\left(F_{Y, Q}\right)_{Q \in \mathcal{Q}}$, e.g. the well-known robust preference of Gilboa and Schmeidler [23]. In the framework of [3, 23], axioms are built on the set of tuples of distributions (e.g. monotonicity is defined with respect to $\mathcal{Q}$-stochastic order) instead of the set of random variables. As a consequence, the set of measures $\mathcal{Q}$ does not play a role in the preference model. This is in sharp contrast to our framework. For instance, Example 3.1 is not allowed as a monotone preference in Gilboa and Schmeidler [23], whereas it is a coherent risk measure in the classic sense of Artzner et al. [4] assuming $2 P \geq Q$. For risk management relevance, it is natural to impose economically relevant axioms on the set of random variables. Later, we shall see that the above discussion plays a significant role in the axiomatic characterisation of scenario-based risk measures.

\subsection{Comonotonic-additive risk measures and Choquet integrals}

As mentioned in Sect. 2.2, the most popular class of risk measures in practice are those that are additive for comonotonic risks. We choose this class as the starting point to establish an axiomatic theory of $\mathcal{Q}$-based risk measures. It is well known that lawbased monetary risk measures are closely related to the notion of Choquet integrals; for instance Yaari's dual utility functionals [43] and Kusuoka representations [31] are based on Choquet integrals.

Definition 3.2 A set function $c: \mathcal{F} \rightarrow \mathbb{R}$ is increasing if $c(A) \leq c(B)$ for $A \subseteq B$, $A, B \in \mathcal{F}$, standard if it is increasing and satisfies $c(\emptyset)=0$ and $c(\Omega)=1$, and $s u b$ modular if

$$
c(A \cup B)+c(A \cap B) \leq c(A)+c(B), \quad A, B \in \mathcal{F} .
$$


Definition 3.3 For a standard set function $c$ and $X \in \mathcal{X}$, the Choquet integral $\int X \mathrm{~d} c$ is defined as

$$
\int X \mathrm{~d} c=\int_{-\infty}^{0}(c(X>x)-1) \mathrm{d} x+\int_{0}^{\infty} c(X>x) \mathrm{d} x .
$$

The integral $\int X \mathrm{~d} c$ in (3.1) might also be well defined on sets larger than the set $\mathcal{X}$ of bounded random variables. Generally, depending on different choices of $c$, one may choose different domains for the Choquet integral. The $Q$-distortion risk measure in (2.3) is exactly a Choquet integral by choosing $c=g \circ Q$.

Now we are ready to present the characterisation for $\mathcal{Q}$-based comonotonicadditive risk measures, which is based on a celebrated result dating back to Schmeidler [40]. Because repeated appearances of some $Q_{1}, \ldots, Q_{n}$ in $\mathcal{Q}$ matter for Theorem 3.4 but not for Definition 2.2, we use both the vector $\underline{Q}$ and set $\mathcal{Q}$.

Theorem 3.4 A risk measure $\rho$ on $\mathcal{X}$ is monetary (resp. coherent), comonotonicadditive and $\mathcal{Q}$-based if and only if

$$
\rho(X)=\int X \mathrm{~d}(\psi \circ \underline{Q}), \quad X \in \mathcal{X},
$$

for some function $\psi:[0,1]^{n} \rightarrow[0,1]$ such that $\psi \circ Q$ is standard (resp. $\psi \circ Q$ is standard and submodular).

Proof Summarising Föllmer and Schied [22, Theorems 4.88 and 4.94], a risk measure $\rho$ on $\mathcal{X}$ is monetary and comonotonic-additive if and only if $\rho$ is a Choquet integral for some standard set function $c$. In addition, $\rho$ is coherent if and only if $c$ is submodular.

Suppose first that $\psi \circ Q$ is a standard set function. Then by the result cited above, the right-hand side of (3.2) defines a comonotonic-additive and monetary risk measure $\rho$. It is coherent if and only if $\psi \circ \underline{Q}$ is additionally submodular. From the definition of $\int X \mathrm{~d}(\psi \circ \underline{Q})$, we have

$$
\rho(X)=\int_{-\infty}^{0}(\psi \circ \underline{Q}[X>x]-1) \mathrm{d} x+\int_{0}^{\infty} \psi \circ \underline{Q}[X>x] \mathrm{d} x,
$$

and hence $\rho$ is $\mathcal{Q}$-based.

Conversely, by the above representation result, $\rho$ can be written as a Choquet integral for some standard set function $c$. If $\rho$ is assumed to be coherent, then $c$ is additionally submodular. By taking $X=\mathbb{1}_{A}, A \in \mathcal{F}$, we have $c(A)=\rho\left(\mathbb{1}_{A}\right)$. Since $\rho$ is $\mathcal{Q}$-based, $\rho\left(\mathbb{1}_{A}\right)$ is determined by the distribution of $\mathbb{1}_{A}$ under $Q_{1}, \ldots, Q_{n}$. Let $R_{\underline{Q}} \subseteq[0,1]^{n}$ be the range of $\underline{Q}$, that is, $R_{\underline{Q}}=\left\{\left(Q_{1}[A], \ldots, Q_{n}[A]\right): A \in \mathcal{F}\right\}$. Since $\left.\rho \overline{(\mathbb{1}}_{A}\right)$ only depends on $\underline{Q}[A]$, we can define $\psi: R_{Q} \rightarrow \mathbb{R}$ by $\psi\left(x_{1}, \ldots, x_{n}\right)=\rho\left(\mathbb{1}_{A}\right)$

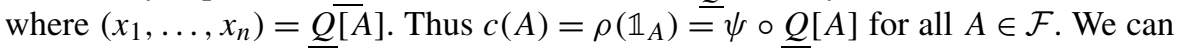
trivially extend the domain of $\psi$ to $[0,1]^{n}$ which does not affect the statement that $c=\psi \circ \underline{Q}$ is standard. 
We refer to a risk measure as in (3.2) as a $Q$-distortion risk measure, which is by Theorem 3.4 precisely a monetary, comonotonic-additive and $\mathcal{Q}$-based risk measure. Coherent $\underline{Q}$-distortion risk measures are referred to as $\underline{Q}$-spectral risk measures. For a $Q$-distortion risk measure $\rho$ as in (3.2), $\psi$ is called its $Q$-distortion function, and it is unique on the range of $Q$ by noting that $\rho\left(\mathbb{1}_{A}\right)=\psi \circ Q[A]$ for all $A \in \mathcal{F}$. It is essential to note that the distortion function of a given risk measure depends on $Q$. For instance, if we take $P, Q \in \mathcal{P}$ and define $\rho(X)=(1 / 3) \mathbb{E}^{P}[X]+(2 / 3) \mathbb{E}^{Q}[X]$, $X \in \mathcal{X}$, then $\rho$ has a $(P, Q)$-distortion function and a $(Q, P)$-distortion function which are different. The classes of $Q$-distortion and $\underline{Q}$-spectral risk measures are the building blocks for the theory of $\mathcal{Q}$-based risk measures.

Clearly, if $n=1$, the concepts of a $\underline{Q}$-distortion risk measure, a $\underline{Q}$-spectral risk measure and a $Q$-distortion function coincide with those defined for a single scenario in Sect. 2.2. In that case, the representation in (3.2) reduces to

$$
\rho(X)=\int X \mathrm{~d}\left(\psi \circ Q_{1}\right), \quad X \in \mathcal{X},
$$

where $\psi \in \mathcal{G}$ (and $\psi \in \mathcal{G}_{\mathrm{cv}}$ if $\rho$ is coherent).

The condition that $\psi \circ Q$ is standard or submodular may be not easy to verify in general, as it involves the joint properties of $\psi$ and $Q$. Next, we establish simple sufficient conditions based solely on $\psi$. These conditions are necessary and sufficient if $Q$ is mutually singular and atomless.

Recall that a function $f:[0,1]^{n} \rightarrow \mathbb{R}$ is called submodular if it holds for all $\mathbf{x}, \mathbf{y} \in[0,1]^{n}$ that $f(\min (\mathbf{x}, \mathbf{y}))+f(\max (\mathbf{x}, \mathbf{y})) \leq f(\mathbf{x})+f(\mathbf{y})$, where $\min (\mathbf{x}, \mathbf{y})$, $\max (\mathbf{x}, \mathbf{y})$ denotes the componentwise minimum and maximum, respectively. By Müller and Stoyan [35, Theorem 3.12.2], the function $f$ is componentwise concave and submodular if and only if for all $\mathbf{x}, \mathbf{y}, \mathbf{w}, \mathbf{z} \in[0,1]^{n}$ with $\mathbf{w} \leq \mathbf{x}, \mathbf{y} \leq \mathbf{z}$ and $\mathbf{w}+\mathbf{z}=\mathbf{x}+\mathbf{y}$, we have

$$
f(\mathbf{x})+f(\mathbf{y}) \geq f(\mathbf{w})+f(\mathbf{z}) .
$$

In addition, if $f$ is two times continuously differentiable, (3.4) holds if and only if all entries of its Hessian are non-positive. We call $f$ increasing if $\mathbf{x} \leq \mathbf{y}$ implies $f(\mathbf{x}) \leq f(\mathbf{y})$.

Proposition 3.5 Let $\psi:[0,1]^{n} \rightarrow[0,1]$ be a function with $\psi(\mathbf{0})=0, \psi(\mathbf{1})=1$.

(i) If $\psi$ is increasing on the range of $Q$, then $\psi \circ Q$ is standard.

(ii) If $\psi$ is increasing, componentwise concave and submodular, then $\psi \circ Q$ is standard and submodular. More precisely, if $\psi$ is increasing and satisfies (3.4) on the range of $\underline{Q}$, then $\psi \circ \underline{Q}$ is standard and submodular.

If $\underline{Q}$ is mutually singular and atomless, then the range of $\underline{Q}$ is $[0,1]^{n}$, and the converses of (i) and (ii) are also true.

Proof Part (i) is trivial. For $A, B \in \mathcal{F}$ and $Q \in \mathcal{Q}$, we always have

$$
Q[A \cup B]+Q[A \cap B]=Q[A]+Q[B]
$$


Therefore, from (3.4), we obtain

$$
\psi \circ \underline{Q}[A \cup B]+\psi \circ \underline{Q}[A \cap B] \leq \psi \circ \underline{Q}[A]+\psi \circ \underline{Q}[B]
$$

which gives the submodularity of $\psi \circ Q$, showing part (ii).

If $Q$ is mutually singular and atomless, the map $Q: \mathcal{F} \rightarrow[0,1]^{n}$ is surjective. Let $A_{1}, \ldots, A_{n} \in \mathcal{F}$ be disjoint sets with $Q_{i}\left[A_{i}\right]=1$ for each $i=1, \ldots, n$. For the converse of part (i), take $\mathbf{x}, \mathbf{y} \in[0,1]^{n}$ with $x_{1} \leq y_{1}$ and $x_{2}=y_{2}, \ldots, x_{n}=y_{n}$. Let $B \in \mathcal{F}$ with $\mathbf{x}=\left(Q_{1}[B], \ldots, Q_{n}[B]\right)$. As $\left(A_{1}, \mathcal{F}, Q_{1}\right)$ is an atomless probability space, there exists a set $C$ with $B \cap A_{1} \subseteq C \subseteq A_{1}$ and $Q_{1}[C]=y_{1}$ (see Delbaen [11, Theorem 1]). We have $\mathbf{y}=\left(Q_{1}[C \cup B], \ldots, Q_{n}[C \cup B]\right)$, which yields the claim. For the converse of part (ii), we show the claim for $n=1$, and the general case follows easily due to the fact that $Q$ is mutually singular. Take $x, y, w, z \in \mathbb{R}$ with $w \leq x, y \leq z$ and $w+z=x+y$. Take $B,\left.C \in \mathcal{F}\right|_{A_{1}}:=\left\{B \cap A_{1}: B \in \mathcal{F}\right\}$, with $Q_{1}[B]=x$ and $Q_{1}[C]=y$. If $Q_{1}[B \cap C]>w$, take $B^{\prime} \subseteq B \backslash C$ with $Q_{1}\left[B^{\prime}\right]=Q_{1}[B \cap C]-w$ and $C^{\prime} \subseteq C \backslash B$ with $Q_{1}\left[C^{\prime}\right]=Q_{1}[B \cap \bar{C}]-w$. Then $\bar{C}=\left(C \backslash C^{\prime}\right) \cup B^{\prime}$ fulfills $Q_{1}[\bar{C}]=y$ and $Q_{1}[B \cap \bar{C}]=w$. If $Q_{1}[B \cap C]<w$, take $B^{\prime} \subseteq(B \cup C)^{c}$ with $Q_{1}\left[B^{\prime}\right]=w-Q_{1}[B \cap C]$ and $C^{\prime} \subseteq C \cap B$ with $Q_{1}\left[C^{\prime}\right]=w-Q_{1}[C \cap B]$. Then $\bar{C}=\left(C \backslash C^{\prime}\right) \cup B^{\prime}$ fulfills $Q_{1}[\bar{C}]=y$ and $Q_{1}[B \cap \bar{C}]=w$. The equation $w+z=x+y=Q_{1}[B]+Q_{1}[\bar{C}]=Q_{1}[B \cap \bar{C}]+Q_{1}[B \cup \bar{C}]$ holds, and hence $z=Q_{1}[B \cup \bar{C}]$. Now the submodularity of $\psi \circ \underline{Q}$ implies (3.4).

Proposition 3.5 implies that it is straightforward to design various comonotonicadditive $\mathcal{Q}$-based risk measures by choosing increasing functions $\psi$. We remark that if $Q$ is not mutually singular, in order for $\psi \circ Q$ to be standard (resp. submodular), it is in general not necessary for $\psi$ to be increasing (resp. componentwise concave and submodular). In Example 3.1, the distortion function of $\rho$ is $\psi:(s, t) \mapsto 2 s-t$, which is not increasing; however, $\rho$ is still a spectral risk measure if $2 P \geq Q$. The following result shows that in this example, $\rho$ cannot be $\mathcal{Q}$-monotone unless the range of $\underline{Q}$ is degenerate in the sense that it has empty interior, which happens if $P=Q$.

Proposition 3.6 Let $\rho$ be a $\underline{Q}$-distortion risk measure with $\underline{Q}$-distortion function $\psi$. The risk measure $\rho$ is $\mathcal{Q}$-monotone if and only if $\psi$ is increasing on the range of $\underline{Q}$.

Proof If $\psi$ is increasing on the range of $\underline{Q}$, the $\mathcal{Q}$-monotonicity of $\rho$ is immediate from (3.3). Conversely, suppose that $\mathbf{x}=\underline{Q}[A] \leq \mathbf{y}=\underline{Q}[B]$ for some $A, B \in \mathcal{F}$. Then $\mathbf{1}_{A} \precsim \stackrel{Q}{\sim} \mathbf{1}_{B}$ for all $Q \in \mathcal{Q}$; hence by $\mathcal{Q}$-monotonicity of $\rho$, we obtain

$$
\psi(\mathbf{x})=\psi \circ \underline{Q}[A]=\rho\left(\mathbf{1}_{A}\right) \leq \rho\left(\mathbf{1}_{B}\right)=\psi \circ \underline{Q}[B]=\psi(\mathbf{y}) .
$$

We proceed to discuss an integral representation of $\underline{Q}$-distortion risk measures. In Sect. 2.2, for a single scenario $Q$, a $Q$-distortion risk measure $\rho_{g}^{Q}$ is defined as

$$
\rho_{g}^{Q}(X)=\int_{-\infty}^{0}(g \circ Q[X>x]-1) \mathrm{d} x+\int_{0}^{\infty} g \circ Q[X>x] d x, \quad X \in \mathcal{X}
$$


If $g$ is left-continuous, then $\rho_{g}^{Q}$ has a Lebesgue integral formulation via an integration by parts (see Dhaene et al. [14, Theorem 6]), that is,

$$
\rho_{g}^{Q}(X)=\int_{0}^{1} \operatorname{VaR}_{p}^{Q}(X) \mathrm{d} \bar{g}(p), \quad X \in \mathcal{X},
$$

where $\bar{g}(t)=1-g(1-t)$ for $t \in[0,1]$. Note that in this case, $\bar{g}$ is right-continuous with $g(0)=1-g(1)=0$; thus $\bar{g}$ is a distribution function on $[0,1]$. This property is key to the integral representation in (3.6). We establish an analogous integral formulation for the case of multiple scenarios under a similar assumption. For a function $\psi:[0,1]^{n} \rightarrow[0,1]$, we define $\bar{\psi}(\mathbf{u})=1-\psi(\mathbf{1}-\mathbf{u}), \mathbf{u} \in[0,1]^{n}$.

Proposition 3.7 Suppose that $\psi:[0,1]^{n} \rightarrow[0,1]$ is such that $\bar{\psi}$ is a distribution function on $[0,1]^{n}$. Let $\rho_{\psi}: \mathcal{X} \rightarrow \mathbb{R}$ be given by

$$
\rho_{\psi}(X)=\int_{[0,1]^{n}} \max \left\{\operatorname{VaR}_{u_{1}}^{Q_{1}}(X), \ldots, \operatorname{VaR}_{u_{n}}^{Q_{n}}(X)\right\} \mathrm{d} \bar{\psi}\left(u_{1}, \ldots, u_{n}\right) .
$$

Then $\rho_{\psi}(X)$ is a $\underline{Q}$-distortion risk measure with $\underline{Q}$-distortion function $\psi$. Moreover, if $\bar{\psi}$ is componentwise convex, then $\rho_{\psi}$ is a $Q$-spectral risk measure.

\section{Proof Let}

$$
Y=\max \left\{F_{X, Q_{1}}^{-1}\left(U_{1}\right), \ldots, F_{X, Q_{n}}^{-1}\left(U_{n}\right)\right\}=\max \left\{\operatorname{VaR}_{U_{1}}^{Q_{1}}(X), \ldots, \operatorname{VaR}_{U_{n}}^{Q_{n}}(X)\right\},
$$

where $\left(U_{1}, \ldots, U_{n}\right) \sim_{\mathbb{P}} \bar{\psi}$. For almost every $x \in \mathbb{R}$, we have

$$
\begin{aligned}
\mathbb{P}[Y \leq x] & =\mathbb{P}\left[F_{X, Q_{1}}^{-1}\left(U_{1}\right) \leq x, \ldots, F_{X, Q_{n}}^{-1}\left(U_{n}\right) \leq x\right] \\
& =\mathbb{P}\left[U_{1} \leq F_{X, Q_{1}}(x), \ldots, U_{n} \leq F_{X, Q_{n}}(x)\right] \\
& =\bar{\psi}\left(Q_{1}[X \leq x], \ldots, Q_{n}[X \leq x]\right)=1-\psi \circ \underline{Q}[X>x] .
\end{aligned}
$$

It follows that

$$
\begin{aligned}
\rho_{\psi}(X) & =\mathbb{E}^{\mathbb{P}}[Y]=\int_{-\infty}^{0}(\mathbb{P}[Y>x]-1) \mathrm{d} x+\int_{0}^{\infty} \mathbb{P}[Y>x] \mathrm{d} x \\
& =\int_{-\infty}^{0}(\psi \circ \underline{Q}[X>x]-1) \mathrm{d} x+\int_{0}^{\infty} \psi \circ \underline{Q}[X>x] \mathrm{d} x=\int X \mathrm{~d}(\psi \circ \underline{Q}) .
\end{aligned}
$$

Note that any distribution function $\bar{\psi}$ is increasing and supermodular. Hence $\psi$ is increasing and submodular, and by Theorem 3.4 and Proposition 3.5, we obtain the desired results.

Proposition 3.7 provides a convenient way to construct various $\underline{Q}$-distortion risk measures. For instance, one may choose $\bar{\psi}$ as an $n$-copula (see $\overline{\mathrm{McN}} \mathrm{Neil}$ et al. [33, Chap. 7]). A direct consequence of Proposition 3.7 is that any $Q$-distortion risk measure with $\underline{Q}$-distortion function $\psi$ has a representation (3.7) if $\bar{\psi}$ is a distribution function. 
For a single scenario $Q$, the distortion function $g$ of a $Q$-spectral risk measure $\rho_{g}^{Q}$ in (3.5) is concave, implying that $\bar{g}$ is automatically a distribution function, and hence $\rho_{g}^{Q}$ always admits a representation as in (3.6). This property does not carry through to the case of $Q$-distortion risk measures in general. More precisely, the $Q$-distortion function of a $\bar{Q}$-spectral risk measure is not necessarily always a distribution function, because all distribution functions on $[0,1]^{n}$ are supermodular, but not vice versa. As a consequence, not all $Q$-spectral risk measures have a representation (3.7). This is in sharp contrast to the case of a single scenario.

\subsection{Coherent risk measures}

As a classic result in the theory of risk measures, the Kusuoka representation [31] states that on an atomless probability space, any single-scenario-based coherent risk measure admits a representation as the supremum over a collection of spectral risk measures, which are in turn mixtures of ES.

One naturally wonders whether a similar result holds true for $\mathcal{Q}$-based coherent risk measures. First, it is straightforward to notice that a supremum over a collection of $Q$-spectral risk measure is always a $\mathcal{Q}$-based coherent risk measure. For the converse direction, we show that a $\mathcal{Q}$-based coherent risk measure admits a representation as the supremum of a collection of mixtures of $Q$-ES for $Q \in \mathcal{Q}$, but this needs a non-trivial condition. More precisely, a $\mathcal{Q}$-mixture of ES is a risk measure $\hat{\rho}$ defined by

$$
\hat{\rho}(X)=\sum_{i=1}^{n} w_{i} \int_{0}^{1} \operatorname{ES}_{p}^{Q_{i}}(X) \mathrm{d} h_{i}(p), \quad X \in \mathcal{X},
$$

for some $\mathbf{w}=\left(w_{1}, \ldots, w_{n}\right) \in[0,1]^{n}$ with $\sum_{i=1}^{n} w_{i}=1$ and distribution functions $h_{1}, \ldots, h_{n}$ on $[0,1]$. Clearly, $\hat{\rho}$ is a $\underline{Q}$-spectral risk measure, as each of the $Q$-ES is. Its $\underline{Q}$-distortion function is given by $\psi(\mathbf{x})=\sum_{i=1}^{n} w_{i} g_{i}\left(x_{i}\right), \mathbf{x} \in[0,1]^{n}$, where $g_{i}$ is for each $i=1, \ldots, n$ the distortion function of $\int_{0}^{1} \operatorname{ES}_{p}^{Q_{i}}(\cdot) \mathrm{d} h_{i}(p)$, and thus

$$
\psi(\mathbf{x})=\sum_{i=1}^{n} w_{i}\left(1-h_{i}\left(1-x_{i}\right)+x_{i} \int_{0}^{1-x_{i}} \frac{1}{1-p} \mathrm{~d} h_{i}(p)\right), \quad \mathbf{x} \in[0,1]^{n} .
$$

We denote by $\Phi_{\mathcal{Q}}$ the set of all $\mathcal{Q}$-mixtures of ES in (3.8). In the next theorem, we establish that if $\underline{Q}$ is mutually singular and atomless, any $\mathcal{Q}$-based coherent risk measure $\rho$ can be written as a supremum of $\mathcal{Q}$-mixtures of ES, namely,

$$
\rho(X)=\sup _{\hat{\rho} \in \Phi} \hat{\rho}(X), \quad X \in \mathcal{X}
$$

for some set $\Phi \subseteq \Phi_{\mathcal{Q}}$. Examples of risk measures of the type (3.9) are discussed in Sect. 4.

Theorem 3.8 (i) If $\rho: \mathcal{X} \rightarrow \mathbb{R}$ is the supremum of some $Q$-spectral risk measures, then it is a $\mathcal{Q}$-based coherent risk measure. 
(ii) If $\underline{Q}$ is mutually singular and atomless, then a risk measure on $\mathcal{X}$ is a $\mathcal{Q}$-based coherent risk measure if and only if it is a supremum of $\mathcal{Q}$-mixtures of ES as in (3.9).

Before proving Theorem 3.8, we establish some auxiliary results which might be of independent interest. First, we discuss the Fatou property (see Delbaen [11]) which we define with respect to a scenario dominating $\mathcal{Q}$. Such a dominating scenario may be chosen as $Q^{*}=(1 / n) \sum_{i=1}^{n} Q_{i}$. Formally, a risk measure $\rho$ is said to satisfy the $\mathcal{Q}$-Fatou property if for any uniformly bounded sequence $X_{1}, X_{2}, \ldots$ in $\mathcal{X}$, the $Q^{*}$-a.s. convergence $X_{k} \stackrel{Q^{*}}{\rightarrow} X \in \mathcal{X}$ implies that $\rho(X) \leq \liminf _{k \rightarrow \infty} \rho\left(X_{k}\right)$. We also introduce on the $Q^{*}$-equivalence classes of $\mathcal{X}$ a norm via $\|\cdot\|_{\mathcal{Q}}=\sup \left\{x>0: Q^{*}[|X|>x]>0\right\}$, which is the usual $L^{\infty}$-norm for essentially bounded random variables on $\left(\Omega, \mathcal{F}, Q^{*}\right)$. Note that in the definitions of the $\mathcal{Q}$-Fatou property and the norm $\|\cdot\|_{\mathcal{Q}}$, the dominating measure $Q^{*}$ can be chosen equivalently as any probability measure dominating $\mathcal{Q}$. It is straightforward to check that all $\mathcal{Q}$-based monetary risk measures are continuous with respect to $\|\cdot\|_{\mathcal{Q}}$. A quasi-convex risk measure $\rho$ is one that satisfies $\rho(\lambda X+(1-\lambda) Y) \leq \max \{\rho(X), \rho(Y)\}$ for all $\lambda \in[0,1]$ and $X, Y \in \mathcal{X}$.

Lemma 3.9 If $Q$ is mutually singular, then any $\mathcal{Q}$-based quasi-convex risk measure that is continuous with respect to $\|\cdot\|_{\mathcal{Q}}$ satisfies the $\mathcal{Q}$-Fatou property.

Proof Write $Q^{*}=(1 / n) \sum_{i=1}^{n} Q_{i}$ and note that $X_{k} \stackrel{Q^{*}}{\rightarrow} X \in \mathcal{X}$ implies $X_{k} \stackrel{Q_{i}}{\rightarrow} X$ for each $i=1, \ldots, n$. We show the lemma in a similar way to Delbaen [12, Theorem 30], which states that a $\left\{Q^{*}\right\}$-based, $\|\cdot\|_{\left\{Q^{*}\right\}}$-continuous and quasi-convex functional satisfies the $\left\{Q^{*}\right\}$-Fatou property (first shown by Jouini et al. [27] with a minor extra condition). A $\mathcal{Q}$-based risk measure is not necessarily $\left\{Q^{*}\right\}$-based, and hence the above result does not directly apply. Nevertheless, we utilise [12, Lemma 11], which gives that for each $i=1, \ldots, n, k \in \mathbb{N}$, there exist a natural number $N_{k}$ and random variables $Z_{k, 1}^{i}, Z_{k, 2}^{i}, \ldots, Z_{k, N_{k}}^{i}$, having the same distribution as $X_{k}$ under $Q_{i}$, such that

$$
\lim _{k \rightarrow \infty} \frac{1}{N_{k}} \sum_{j=1}^{N_{k}} Z_{k, j}^{i}=X \quad \text { in }\|\cdot\|_{\left\{Q_{i}\right\}} .
$$

The numbers $N_{k}$ can be chosen independently of $i$, as explained by Delbaen [12, Remark 40]. For $k \in \mathbb{N}$ and $j=1, \ldots, N_{k}$, let $Y_{k, j}=\sum_{i=1}^{n} Z_{k, j}^{i} \mathbb{1}_{A_{i}}$, where $A_{1}, \ldots, A_{n} \in \mathcal{F}$ are disjoint sets such that $Q_{i}\left[A_{i}\right]=1$ for $i=1, \ldots, n$. It is clear that for each choice of $(i, j, k), Y_{k, j}$ has the same distribution as $X_{k}$ under $Q_{i}$, and

$$
\lim _{k \rightarrow \infty} \frac{1}{N_{k}} \sum_{j=1}^{N_{k}} Y_{k, j}=X \quad \text { in }\|\cdot\|_{\mathcal{Q}} .
$$


Therefore, $\rho\left(Y_{k, j}\right)=\rho\left(X_{k}\right)$. Finally, as $\rho$ is $\|\cdot\|_{\mathcal{Q}}$-continuous, quasi-convex and $\mathcal{Q}$-based, we have

$$
\rho(X)=\lim _{k \rightarrow \infty} \rho\left(\frac{1}{N_{k}} \sum_{j=1}^{N_{k}} Y_{k, j}\right) \leq \liminf _{k \rightarrow \infty} \max _{j=1, \ldots, N_{k}} \rho\left(Y_{k, j}\right)=\liminf _{k \rightarrow \infty} \rho\left(X_{k}\right) .
$$

Thus $\rho$ satisfies the $\mathcal{Q}$-Fatou property.

As a direct consequence of Lemma 3.9 , if $\mathcal{Q}$ is mutually singular, then any $\mathcal{Q}$-based coherent risk measure, such as a $Q$-spectral risk measure, satisfies the $\mathcal{Q}$-Fatou property. Next, we present a lemma which serves as a building block for the proof of Theorem 3.8. For $X \in \mathcal{X}$, let

$$
L_{X}(\mathcal{Q})=\{Y \in \mathcal{X}: Y \stackrel{\mathrm{d}}{=} Q X \text { for all } Q \in \mathcal{Q}\}
$$

be the set of all random variables identically distributed as $X$ under each measure in $\mathcal{Q}$. Clearly, $X \in L_{X}(\mathcal{Q})$ and hence $L_{X}(\mathcal{Q})$ is not empty.

Lemma 3.10 Suppose $\underline{Q}$ is mutually singular and atomless and $P \ll \frac{1}{n} \sum_{i=1}^{n} Q_{i}$. Then the functional $\rho: \overline{\mathcal{X}} \rightarrow \mathbb{R}, \rho(X)=\sup _{Y \in L_{X}(\mathcal{Q})} \mathbb{E}^{P}[Y]$, is a $\mathcal{Q}$-mixture of ES.

Proof Let $A_{1}, \ldots, A_{n} \in \mathcal{F}$ be disjoint sets with $Q_{i}\left[A_{i}\right]=1$ for $i=1, \ldots, n$. Write $Q^{*}=\frac{1}{n} \sum_{i=1}^{n} Q_{i}$ and $Z=\mathrm{d} P / \mathrm{d} Q^{*}$. For each $i=1, \ldots, n$, let $U_{i}$ be under $Q_{i}$ a uniform random variable on $[0,1]$ such that $Z=F_{Z, Q_{i}}^{-1}\left(U_{i}\right) Q_{i}$-almost surely. The existence of such a random variable $U_{i}$ can be guaranteed for instance by Föllmer and Schied [22, Lemma A.32]. By the Fréchet-Hoeffding inequality (see Rüschendorf $\left[39\right.$, Remark 3.25]), we have $\mathbb{E}^{Q_{i}}[Z Y] \leq \mathbb{E}^{Q_{i}}\left[Z F_{Y, Q_{i}}^{-1}\left(U_{i}\right)\right]$ for $Y \in \mathcal{X}$. It follows that for $Y \in L_{X}(\mathcal{Q})$,

$$
\mathbb{E}^{P}[Y]=\frac{1}{n} \sum_{i=1}^{n} \mathbb{E}^{Q_{i}}\left[\frac{\mathrm{d} P}{\mathrm{~d} Q^{*}} Y\right] \leq \frac{1}{n} \sum_{i=1}^{n} \mathbb{E}^{Q_{i}}\left[Z F_{X, Q_{i}}^{-1}\left(U_{i}\right)\right] .
$$

On the other hand, it is easy to verify that $\sum_{i=1}^{n} F_{X, Q_{i}}^{-1}\left(U_{i}\right) \mathbb{1}_{A_{i}} \in L_{X}(\mathcal{Q})$ and

$$
\mathbb{E}^{P}\left[\sum_{i=1}^{n} F_{X, Q_{i}}^{-1}\left(U_{i}\right) \mathbb{1}_{A_{i}}\right]=\frac{1}{n} \sum_{i=1}^{n} \mathbb{E}^{Q_{i}}\left[Z F_{X, Q_{i}}^{-1}\left(U_{i}\right)\right] \text {. }
$$

Therefore

$$
\sup _{Y \in L_{X}(\mathcal{Q})} \mathbb{E}^{P}[Y]=\frac{1}{n} \sum_{i=1}^{n} \mathbb{E}^{Q_{i}}\left[Z F_{X, Q_{i}}^{-1}\left(U_{i}\right)\right]
$$

Note that

$$
\mathbb{E}^{Q_{i}}\left[Z F_{X, Q_{i}}^{-1}\left(U_{i}\right)\right]=\int_{0}^{1} F_{Z, Q_{i}}^{-1}(u) F_{X, Q_{i}}^{-1}(u) \mathrm{d} u,
$$


and the function $\bar{g}:[0,1] \rightarrow[0,1], t \mapsto \int_{0}^{t} F_{Z, Q_{i}}^{-1}(u) \mathrm{d} u$, is in $\mathcal{G}$ and convex. It follows that the mapping $X \mapsto \mathbb{E}^{Q_{i}}\left[Z F_{X, Q_{i}}^{-1}\left(U_{i}\right)\right]$ is a spectral risk measure of the form (3.6). Therefore, $\rho$ is a linear combination of $Q$-spectral risk measures with $Q \in \mathcal{Q}$. Note that each $Q$-spectral risk measure is a mixture of $Q$-ES (see Kusuoka [31, Theorem 4$]$ ), and hence $\rho$ is a $\mathcal{Q}$-mixture of ES.

Proof of Theorem 3.8 Concerning part (i), it suffices to observe that $Q$-spectral risk measures are coherent, and that a supremum of $\mathcal{Q}$-based coherent risk measures is also a $\mathcal{Q}$-based coherent risk measure.

For part (ii), since $\rho$ is coherent, it has by Lemma 3.9 the $\mathcal{Q}$-Fatou property. From the classic coherent risk measure representation (see Delbaen [11]), there exists a set $\mathcal{R} \subseteq \mathcal{P}$ of probability measures, absolutely continuous with respect to $Q^{*}$, such that

$$
\rho(X)=\sup _{P \in \mathcal{R}} \mathbb{E}^{P}[X], \quad X \in \mathcal{X} .
$$

Now fix $X \in \mathcal{X}$. As $\rho$ is $\mathcal{Q}$-based, $\rho(Y)=\rho(X)$ for all $Y \in L_{X}(\mathcal{Q})$. It follows that

$$
\rho(X)=\sup _{Y \in L_{X}(\mathcal{Q})} \sup _{P \in \mathcal{R}} \mathbb{E}^{P}[Y]=\sup _{P \in \mathcal{R}} \sup _{Y \in L_{X}(\mathcal{Q})} \mathbb{E}^{P}[Y] .
$$

By Lemma 3.10, for each $P \in \mathcal{R}$, the map $\mathcal{X} \rightarrow \mathbb{R}, X \mapsto \sup _{Y \in L_{X}(\mathcal{Q})} \mathbb{E}^{P}[Y]$, is a mixture of $Q$-ES for $Q \in \mathcal{Q}$. Therefore, $\rho$ is the supremum of $\mathcal{Q}$-mixtures of ES.

The representation in (3.9) resembles the risk measure in the Basel FRTB formula; see Sect. 1. Indeed, it is remarkable that only using maxima and linear combinations of $Q$-ES, as done in [5], one arrives at all possible $\mathcal{Q}$-based coherent risk measures if $\mathcal{Q}$ is mutually singular. Certainly, the set of measures $\mathcal{Q}$ chosen in [5] is not necessarily mutually singular, allowing more possible forms of coherent risk measures. Nevertheless, the maximum of linear combinations of $Q$-ES is the only form that is coherent for all choices of scenarios, among the general class of scenario-based risk measures.

The characterisation in Theorem 3.8 on the set of bounded random variables can be extended to general $L^{q}$-spaces. In the following, let $Q_{0} \in \mathcal{P}$ be a probability measure dominating $\mathcal{Q}$ and $L^{q}\left(Q_{0}\right), q \geq 1$, the set of equivalence classes of random variables with a finite $q$-th moment under $Q_{0}$.

Proposition 3.11 Suppose that $Q$ is mutually singular and atomless and $q \geq 1$. Then

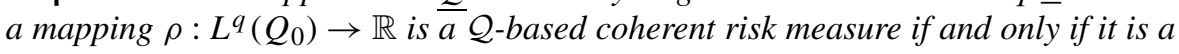
supremum of $\mathcal{Q}$-mixtures of ES as in (3.9).

Proof Clearly, the supremum of $\mathcal{Q}$-mixtures of ES is a $\mathcal{Q}$-based coherent risk measure. So it suffices to show the "only if" statement. First, is it easy to verify, with the same arguments as in Theorem 3.8, that Lemma 3.10 holds true with " $\mathcal{X} \rightarrow \mathbb{R}$ " replaced by " $L^{q}\left(Q_{0}\right) \rightarrow \mathbb{R} \cup\{\infty\}$ ". Note that $\rho$ in Proposition 3.11 is finite-valued and all finite-valued convex risk measures on $L^{q}$ are continuous (see Rüschendorf [39, Theorem 7.24]). Therefore $\rho$ admits a representation in (3.10) with $\mathcal{X}$ replaced by $L^{q}\left(Q_{0}\right)$. Using the same argument in the proof of Theorem 3.8 (ii), we conclude that $\rho$ is the supremum of $\mathcal{Q}$-mixtures of ES. 
Remark 3.12 In Proposition 3.11, we have formulated $\rho$ such that it is real-valued on $L^{q}\left(Q_{0}\right)$. This assumption is useful as not all $\mathcal{Q}$-mixtures of ES are finite on $L^{q}\left(Q_{0}\right)$, and this is because integrability is not preserved among equivalent measures.

Theorem 3.8 is the most technical result of this paper. The mutual singularity of $Q$ is used repeatedly in the proof and is indispensable for part (ii). Without it, the converse of Theorem 3.8 (ii) is not true. Note that the representation (3.9) is $\mathcal{Q}$-monotone, and we have seen in Example 3.1 that there are $\mathcal{Q}$-based coherent risk measures which are not $\mathcal{Q}$-monotone; see also Proposition 3.6.

We expect that an interesting characterisation result for $\mathcal{Q}$-based coherent risk measures without the assumption of mutual singularity of $Q$ will at least require the additional assumption of $\rho$ being $\mathcal{Q}$-monotone, possibly even for a weaker stochastic order than the usual stochastic ordering. However, even with such an extra assumption, the characterisation problem without assuming mutual singularity is wide open and seems beyond the reach of current technical tools. The current line of proof requires the understanding of the functional defined in Lemma 3.10. That functional is easy to understand via the Hardy-Littlewood inequality in the case of mutual singularity. However, without this assumption, the functional is hard to understand and need not be the right tool for a characterisation result.

Remark 3.13 Kou and Peng [28] considered $\mathcal{Q}$-based risk measures $\rho$ with the representation $\rho(X)=f\left(\rho_{1}(X), \ldots, \rho_{n}(X)\right)$ for some aggregation function $f: \mathbb{R}^{n} \rightarrow \mathbb{R}$ and risk measures $\rho_{1}, \ldots, \rho_{n}$ each based on one scenario; see [28, Eq. (22)]. They imposed some axioms on $f$ and $\rho_{1}, \ldots, \rho_{n}$. Our axioms are directly imposed on the risk measure $\rho$ and we do not assume a particular functional form.

\section{Scenario-based VaR and ES, and other examples}

Because of the prominent importance of VaR and ES in external regulatory capital calculation and internal risk management, we investigate several examples of scenario-based risk measures which can be seen as natural generalisations of VaR and ES in a multi-scenario framework. In this section, $\mathcal{Y}$ is any convex cone of random variables containing $\mathcal{X}$.

Example 4.1 Let $p \in(0,1)$. For a collection of measures $\mathcal{Q}$, the Max-ES (MES) is defined as

$$
\operatorname{MES}_{p}^{\mathcal{Q}}(X)=\sup _{Q \in \mathcal{Q}} \operatorname{ES}_{p}^{Q}(X), \quad X \in \mathcal{Y},
$$

and the Max-VaR (MVaR) as

$$
\operatorname{MVaR}_{p}^{\mathcal{Q}}(X)=\sup _{Q \in \mathcal{Q}} \operatorname{VaR}_{p}^{Q}(X), \quad X \in \mathcal{Y} .
$$

Max-ES and Max-VaR incorporate information evaluated under each scenario and make a conservative capital calculation by taking the maximum. We call them maxtype risk measures. They appear in the literature on robust optimisation; see e.g. Zhu 
and Fukushima [44], Zymler et al. [45]. Similarly to the single-scenario-based ES and VaR in (2.1) and (2.2), Max-ES and Max-VaR have different mathematical properties.

Max-ES is known to be coherent (Zhu and Fukushima [44]); but in general, and in contrast to the single-scenario-based ES, it fails to be comonotonic-additive, see Example A.1 in Appendix A.1. Quite surprisingly, the risk measure $\operatorname{MVaR}_{p}^{\mathcal{Q}}$ satisfies comonotonic-additivity. If $\mathcal{Q}$ is finite with $n$ elements, this follows by choosing $\bar{\psi}$ as the distribution function of the Dirac measure at $(p, \ldots, p) \in[0,1]^{n}$ in Proposition 3.7. For general sets $\mathcal{Q}$ of scenarios, see Appendix A.2.

Remark 4.2 As a classic result (Delbaen [11]), a coherent risk measure $\rho$ on $\mathcal{X}$ with the Fatou property has a dual representation

$$
\rho(X)=\sup _{Q \in \mathcal{Q}} \mathbb{E}^{Q}[X], \quad X \in \mathcal{X},
$$

for some set of probability measures $\mathcal{Q}$. Clearly, $\rho$ is a max-type $\mathcal{Q}$-based risk measure with a different building block (the expectation) than Max-VaR and Max-ES. This includes in particular the methodology for margin requirement calculation developed by the Chicago Mercantile Exchange [33, p. 63]. In addition, Duffie et al. [16] feature max-type risk measures in the context of calculation of initial margins for bilateral portfolios.

Other than the max-type, using a finite or continuous mixture is also a convenient and simple way to construct $\mathcal{Q}$-based risk measures (however, using an infinite mixture requires a measure specified on $\mathcal{Q}$, which is not always easy). Below we present a few other ways to formulate ES in the framework of $\mathcal{Q}$-based risk measures. One can also define corresponding versions of VaR or any other law-based risk measure, but we take ES as a main example in this section due to its relevance in Basel III \& IV.

Example 4.3 Take $p \in(0,1)$ and a finite $\mathcal{Q}=\left\{Q_{1}, \ldots, Q_{n}\right\}$, and define the Average$E S(A E S)$ as the average of ES across different scenarios, that is,

$$
\operatorname{AES}_{p}^{\mathcal{Q}}(X)=\frac{1}{n} \sum_{i=1}^{n} \operatorname{ES}_{p}^{Q_{i}}(X), \quad X \in \mathcal{Y} .
$$

It is obvious that $\operatorname{AES}_{p}^{\mathcal{Q}}$ is a coherent and comonotonic-additive risk measure. Its $\underline{Q}$-distortion function $\psi(\mathbf{u})=(n(1-p))^{-1} \sum_{i=1}^{n} \min \left\{u_{i}, 1-p\right\}$ is increasing, componentwise concave and submodular. In the case $n=2$, the associated function $\bar{\psi}(\mathbf{u})=1-\psi(1-\mathbf{u}), \mathbf{u} \in[0,1]^{n}$, is a componentwise convex distribution function on $[0,1]^{2}$; so in this case, $\operatorname{AES}_{p}^{\mathcal{Q}}$ has an integral representation by Proposition 3.7. For $n \geq 3, \bar{\psi}$ fails to be a distribution function.

Example 4.4 Recall that the single-scenario-based ES in (2.2) is an average of VaR of probability level beyond $p \in(0,1)$. Utilising this connection, we define the integral $\operatorname{Max}$-ES (iMES) as the integral of MVaR, that is, 


$$
\operatorname{iMES}_{p}^{\mathcal{Q}}(X)=\frac{1}{1-p} \int_{p}^{1} \operatorname{MVaR}_{q}^{\mathcal{Q}}(X) \mathrm{d} q, \quad X \in \mathcal{Y} .
$$

For finite $\mathcal{Q}$ with $n$ elements, we can choose $\bar{\psi}$ as the distribution function of a uniform distribution over the diagonal line segment

$$
\left\{\left(u_{1}, \ldots, u_{n}\right) \in[p, 1]^{n}: u_{1}=u_{2}=\cdots=u_{n}\right\}
$$

to obtain the integral Max-ES as a special case of Proposition 3.7. Its $Q$-distortion function $\psi$ is given by $\psi(\mathbf{u})=\min \left\{\max \left\{u_{1}, \ldots, u_{n}\right\}, 1-p\right\} /(1-p), \mathbf{u} \in[0,1]^{n}$. This verifies that $\operatorname{MES}_{p}^{\mathcal{Q}}$ is comonotonic-additive. However, $\psi$ is not componentwise concave, which implies that $\operatorname{iMES}_{p}^{\mathcal{Q}}$ is not a coherent risk measure for mutually singular $\underline{Q}$ by Proposition 3.5. For random variables $X$ such that $F_{X, Q_{i}}, i=1, \ldots, n$, are stochastically ordered, it holds that $\operatorname{iMES}_{p}^{\mathcal{Q}}(X)=\operatorname{MES}_{p}^{\mathcal{Q}}(X)$.

Noting that $\operatorname{MVaR}_{q}^{\mathcal{Q}}(X)$ is increasing in $q$, one may equivalently write

$$
\operatorname{iMES}_{p}^{\mathcal{Q}}(X)=\operatorname{ES}_{p}^{\mathbb{P}}\left(\operatorname{MVaR}_{U}^{\mathcal{Q}}(X)\right)=\operatorname{ES}_{p}^{\mathbb{P}}\left(\sup _{Q \in \mathcal{Q}} F_{X, Q}^{-1}(U)\right), \quad X \in \mathcal{Y}
$$

where $U \sim_{\mathbb{P}} \mathrm{U}[0,1]$.

Example 4.5 Another way to utilise the ES and a maximum operator is via independent replications of $X$ under different scenarios. Let $p \in(0,1), Q_{1}, \ldots, Q_{n}$ be distinct scenarios and $\mathcal{Q}=\left\{Q_{1}, \ldots, Q_{n}\right\}$. Define a replicated Max-ES (rMES) as the ES of a maximum of independent copies, that is,

$$
\operatorname{rMES}_{p}^{\mathcal{Q}}(X)=\operatorname{ES}_{p}^{\mathbb{P}}\left(\max _{i=1, \ldots, n} X_{i}\right), \quad X \in \mathcal{Y}
$$

where $X_{i} \sim_{\mathbb{P}} F_{X, Q_{i}}, i=1, \ldots, n$, and $X_{1}, \ldots, X_{n}$ are independent under $\mathbb{P}$. The risk measure $\operatorname{rMES}_{p}^{\mathcal{Q}}$ is defined for a finite collection $\mathcal{Q}$ so that the maximum in (4.5) is well posed. It has some similarity to MINVAR in Cherny and Madan [10]; see Example 4.9 for more details. The replicated Max-ES grows to the maximum of the essential supremum of the distributions $F_{X, Q_{i}}$ when the number $n$ of scenarios goes to infinity. Therefore, it is likely to be too conservative or even plainly uninformative if $n$ is too large; see also Sect. 5 .

The replicated Max-ES is comonotonic-additive and coherent. We can determine its $Q$-distortion function as follows. Suppose that $X_{1}, \ldots, X_{n}$ are independent under $\mathbb{P}, \bar{X}_{i} \sim_{\mathbb{P}} F_{X, Q_{i}}, i=1, \ldots, n$, and $U \sim_{\mathbb{P}} \mathrm{U}[p, 1]$. The distribution function $F_{\max }$ of $\max \left\{X_{1}, \ldots, X_{n}\right\}$ under $\mathbb{P}$ is $x \mapsto \prod_{i=1}^{n} Q_{i}[X \leq x]$. Moreover, the survival function of $F_{\max }^{-1}(U)$ under $\mathbb{P}$ is given by 


$$
\begin{aligned}
x \mapsto 1-\frac{\left(F_{\max }(x)-p\right)^{+}}{1-p} & =1-\frac{\left(\prod_{i=1}^{n} Q_{i}[X \leq x]-p\right)^{+}}{1-p} \\
& =\frac{\min \left\{1-\prod_{i=1}^{n} Q_{i}[X \leq x], 1\right\}}{1-p} .
\end{aligned}
$$

Hence by letting $\psi:[0,1]^{n} \rightarrow[0,1], \mathbf{u} \mapsto \min \left\{1-\prod_{i=1}^{n}\left(1-u_{i}\right), 1-p\right\} /(1-p)$, we have $\psi \circ \underline{Q}[X>x]=\mathbb{P}\left[F_{\max }^{-1}(U)>x\right], x \in \mathbb{R}$, and hence

$$
\int X \mathrm{~d}(\psi \circ \underline{Q})=\mathbb{E}^{\mathbb{P}}\left[F_{\max }^{-1}(U)\right]=\operatorname{ES}_{p}^{\mathbb{P}}\left(\max _{i=1, \ldots, n} X_{i}\right)=\operatorname{rMES}_{p}^{\mathcal{Q}}(X) .
$$

Thus $\psi$ is the $\underline{Q}$-distortion function of $\operatorname{iMES}_{p}^{\mathcal{Q}}$ which is componentwise concave and submodular.

We find that $\bar{\psi}(\mathbf{u})=\left(\prod_{i=1}^{n} u_{i}-p\right)^{+} /(1-p), \mathbf{u} \in[0,1]^{n}$, is a distribution function; so the replicated Max-ES has an integral representation as in Proposition 3.7. Indeed, $\bar{\psi}$ arises when combining uniformly distributed marginals on $[p, 1]$ with an Archimedean copula with generator

$$
[0,1] \rightarrow[0, \infty), \quad t \mapsto-\log ((1-p) t+p) .
$$

This generator is completely monotone; so we obtain a valid copula for any dimension $n$ (see McNeil and Nešlehová [34]).

The risk measures $\operatorname{iMES}_{p}^{\mathcal{Q}}$, and $\operatorname{rMES}_{p}^{\mathcal{Q}}$ are connected through the fact that if $X_{1}, \ldots, X_{n}$ in (4.5) are comonotonic instead of independent under $\mathbb{P}$, then (4.5) gives rise to (4.4). Each of the $\mathcal{Q}$-based risk measures $\operatorname{MES}_{p}^{\mathcal{Q}}, \operatorname{AES}_{p}^{\mathcal{Q}}, \operatorname{iMES}_{p}^{\mathcal{Q}}$ and $\operatorname{rMES}_{p}^{\mathcal{Q}}$ may be seen as a natural generalisation of the single-scenario-based risk measure $\mathrm{ES}_{p}^{Q}$. Although using similar ideas, these risk measures have different properties and values. If $\mathcal{Q}=\{Q\}$, the above five risk measures are all equal. They are in general non-equivalent and satisfy an order summarised below. Proposition 4.6 summarises the results of this section; some are already explained above.

Proposition 4.6 Let $\mathcal{Q}$ be a finite collection of scenarios and $p \in(0,1)$.

(i) $\operatorname{MES}_{p}^{\mathcal{Q}}$ is coherent, but in general not comonotonic-additive.

(ii) $\mathrm{MVaR}_{p}^{\mathcal{Q}}$ is comonotonic-additive, positively homogeneous and monetary, but in general not coherent.

(iii) $\operatorname{AES}_{p}^{\mathcal{Q}}$ is comonotonic-additive and coherent.

(iv) $\mathrm{iMES}_{p}^{\mathcal{Q}}$ is comonotonic-additive, but in general not coherent.

(v) $\operatorname{rMES}{ }_{p}^{\mathcal{Q}}$ is comonotonic-additive and coherent.

(vi) $\operatorname{AES}_{p}^{\mathcal{Q}}(X) \leq \operatorname{MES}_{p}^{\mathcal{Q}}(X) \leq \operatorname{iMES}_{p}^{\mathcal{Q}}(X) \leq \operatorname{rMES}_{p}^{\mathcal{Q}}(X)$ for all $X \in \mathcal{Y}$.

(vii) If $\mathcal{Q}$ is a singleton, all inequalities in (vi) are equalities.

Proof It only remains to show (vi). The first inequality is trivial. For the second, observe that for each $Q \in \mathcal{Q}$ and $U \sim_{\mathbb{P}} \mathrm{U}[0,1]$, we have $\operatorname{ES}_{p}^{\mathcal{Q}}(X)=\operatorname{ES}_{p}^{\mathbb{P}}\left(F_{X, Q}^{-1}(U)\right)$; hence the claim follows by using (4.4). The third inequality follows by observing 
that the $Q$-distortion function of iMES is pointwise smaller than the $Q$-distortion function of rMES. This holds since $\max \left\{u_{1}, \ldots, u_{n}\right\} \leq 1-\prod_{i=1}^{n}\left(1-u_{i}\right)$ is equivalent to $\prod_{i=1}^{n}\left(1-u_{i}\right) \leq\left(1-u_{j}\right)$ for all $j=1, \ldots, n$, where the latter clearly holds for any $\mathbf{u} \in[0,1]^{n}$.

The above examples illustrate that the framework of $\mathcal{Q}$-based risk measures is flexible and allows a great variety of risk measures to be formulated, even simply from ES and a fixed $p$. Parts (i), (ii) and (iv) of Proposition 4.6 also hold true if $\mathcal{Q}$ is infinite; see Appendix A.2.

There is a simple relationship between iMES (MVaR) and ES (VaR) when the collection of scenarios $\mathcal{Q}$ consists of the economic scenarios in Example 2.1.

Proposition 4.7 Let $\mathcal{Q}=\left\{Q_{\theta}: \theta \in K\right\}$ be as in Example 2.1. For $p \in(0,1)$, we have $\operatorname{MVaR}_{p}^{\mathcal{Q}}(X) \geq \operatorname{VaR}_{p}^{\mathbb{P}}(X)$ and $\operatorname{iMES}_{p}^{\mathcal{Q}}(X) \geq \operatorname{ES}_{p}^{\mathbb{P}}(X)$ for all $X \in \mathcal{Y}$.

Proof We show $\operatorname{MVaR}_{p}^{\mathcal{Q}}(X) \geq \operatorname{VaR}_{p}^{\mathbb{P}}(X)$, which implies $\operatorname{iMES}_{p}^{\mathcal{Q}}(X) \geq \operatorname{ES}_{p}^{\mathbb{P}}(X)$. Take $x<\operatorname{VaR}_{p}^{\mathbb{P}}(X)$ so that $\mathbb{P}[X \leq x]<p$. As $\mathbb{P}$ is a convex combination of $Q_{\theta}, \theta \in K$, we must have $Q_{\theta}[X \leq x]<p$ for some $\theta \in K$, which implies that $x \leq \operatorname{VaR}_{p}^{Q_{\theta}}(X) \leq \operatorname{MVaR}_{p}^{\mathcal{Q}}(X)$. Therefore,

$$
\operatorname{MVaR}_{p}^{\mathcal{Q}}(X) \geq \sup \left\{x \in \mathbb{R}: x<\operatorname{VaR}_{p}^{\mathbb{P}}(X)\right\}=\operatorname{VaR}_{p}^{\mathbb{P}}(X) .
$$

Proposition 4.7 implies that when using the economic scenarios in Example 2.1, iMES (MVaR) is more conservative than ES (VaR) over the unconditional real-world probability measure $\mathbb{P}$. Note that $\operatorname{MES}_{p}^{\mathcal{Q}}(X) \geq \operatorname{ES}_{p}^{\mathbb{P}}(X)$ does not hold in general (see Example A.2 in Appendix A.1 for a counterexample), although this inequality almost always holds empirically, as we shall see in the data analysis in Sect. 5.

Below, we discuss two more connections of our risk measures to the ones in the literature.

Example 4.8 Recently, Righi [37] studied combinations of risk measures. For a finite collection of scenarios $\left\{Q_{1}, \ldots, Q_{n}\right\}$, these take the form $f\left(\rho^{Q_{1}}, \ldots, \rho^{Q_{n}}\right)$ for some function $f$, where $\rho Q_{i}$ is $\left\{Q_{i}\right\}$-based, $i=1, \ldots, n$, and this includes the max-type risk measures. Another scenario-based risk measure of the type $f\left(\rho^{Q_{1}}, \ldots, \rho^{Q_{n}}\right)$ is given by Kou and Peng [28], defined as

$$
\rho(X)=\sup _{\left(w_{1}, \ldots, w_{n}\right) \in \mathcal{W}} \sum_{i=1}^{n} w_{i} \rho_{h_{i}}^{Q_{i}}(X), \quad X \in \mathcal{X},
$$

where $\rho_{h_{i}}^{Q_{i}}, i=1, \ldots, n$, are $Q_{i}$-distortion risk measures as in (2.3) and $\mathcal{W}$ is a subset of the standard simplex $\left\{\left(w_{1}, \ldots, w_{n}\right) \in[0,1]^{n}: \sum_{i=1}^{n} w_{i}=1\right\}$.

Example 4.9 In (3.7), by choosing $\bar{\psi}(\mathbf{u})=\prod_{i=1}^{n} u_{i}$ for $\mathbf{u} \in[0,1]^{n}$, we obtain

$$
\rho(X)=\mathbb{E}^{\mathbb{P}}\left[\max \left\{X_{1}, \ldots, X_{n}\right\}\right], \quad X \in \mathcal{X},
$$


where $F_{X_{i}, \mathbb{P}}=F_{X, Q_{i}}$ for $i=1, \ldots, n$ and $X_{1}, \ldots, X_{n}$ are independent under $\mathbb{P}$. Then $\rho$ is a $Q$-spectral risk measure with corresponding $Q$-distortion function $\psi(\mathbf{u})=1-\prod_{i=1}^{n}\left(1-u_{i}\right), \mathbf{u} \in[0,1]^{n}$. The risk measure $\rho$ is coherent. The singlescenario-based risk measure MINVAR (see Cherny and Madan [10]) defined as

$$
\operatorname{MinVAR}(X)=\mathbb{E}^{\mathbb{P}}\left[\max \left\{X_{1}, \ldots, X_{n}\right\}\right], \quad X \in \mathcal{X},
$$

where $X_{1}, \ldots, X_{n}$ are i.i.d. copies of $X$ under $\mathbb{P}$, is a special case of $\rho$ by choosing $Q_{1}=\cdots=Q_{n}=\mathbb{P}$.

\section{Data analysis for scenario-based risk measures}

In this section, we discuss two examples of data analysis for $\mathcal{Q}$-based risk measures. The two examples are conceptually different with the aim to illustrate different possible interpretations for the collection $\mathcal{Q}$ of scenarios; cf. Remark 2.4. Various versions of the $\mathcal{Q}$-based expected shortfalls as in Sect. 4 are chosen to illustrate the main ideas; clearly, the analysis may be applied to other scenario-based risk measures.

\subsection{Max-expected shortfalls for economic scenarios}

Taking up Example 2.1, we consider $Q_{i}=\mathbb{P}\left[\cdot \mid \Theta=\theta_{i}\right], i=1, \ldots, 4$, where $\Theta$ is an economic factor taking values in $\left\{\theta_{1}, \ldots, \theta_{4}\right\}$ and $\mathbb{P}$ can be interpreted as the realworld probability measure. While the $\mathcal{Q}$-based expected shortfalls of $X$ are clearly defined mathematical quantities, it is not completely obvious how to estimate them. The approach we describe can be justified under suitable assumptions on the datagenerating processes. However, we leave a detailed study of the proposed estimator for future work.

Here, we assume that we have four data sets

$$
D_{1}=\left\{X_{1}^{Q_{1}}, \ldots, X_{N_{1}}^{Q_{1}}\right\}, \ldots, D_{4}=\left\{X_{1}^{Q_{4}}, \ldots, X_{N_{4}}^{Q_{4}}\right\}
$$

with the property that the empirical distribution of $D_{i}$ is a reasonable estimate of $F_{X, Q_{i}}, i=1, \ldots, 4$. Then we estimate the risk measures $\mathrm{ES}_{p}, \mathrm{MES}_{p}, \mathrm{iMES}_{p}$ and rMES $_{p}$ given by (4.1), (4.2), (4.3) and (4.5), respectively, by their empirical counterparts.

Given a series of returns $\left(X_{t}\right)_{t \in \mathbb{N}}$, we compute for each trading day $\mathcal{Q}$-based expected shortfalls of $X_{t}$ estimated on a rolling window of length 250 . The four scenarios can be interpreted as

$$
\left\{\theta_{1}, \ldots, \theta_{4}\right\}=\{\text { high volatility, low volatility }\} \times\{\text { good economy, bad economy }\}
$$

The value of $\Theta$ is based on the values of VIX (high volatility/low volatility) and S\&P 500 (good economy/bad economy). To be precise, for day $t_{0}$, we use the time window $t_{0}-250, \ldots, t_{0}-1$. Then we use the VIX to split the time period into two categories depending on whether the VIX is higher or lower than its empirical median in the time window. We removed a log-linear trend from the S\&P 500 since 1950, and 


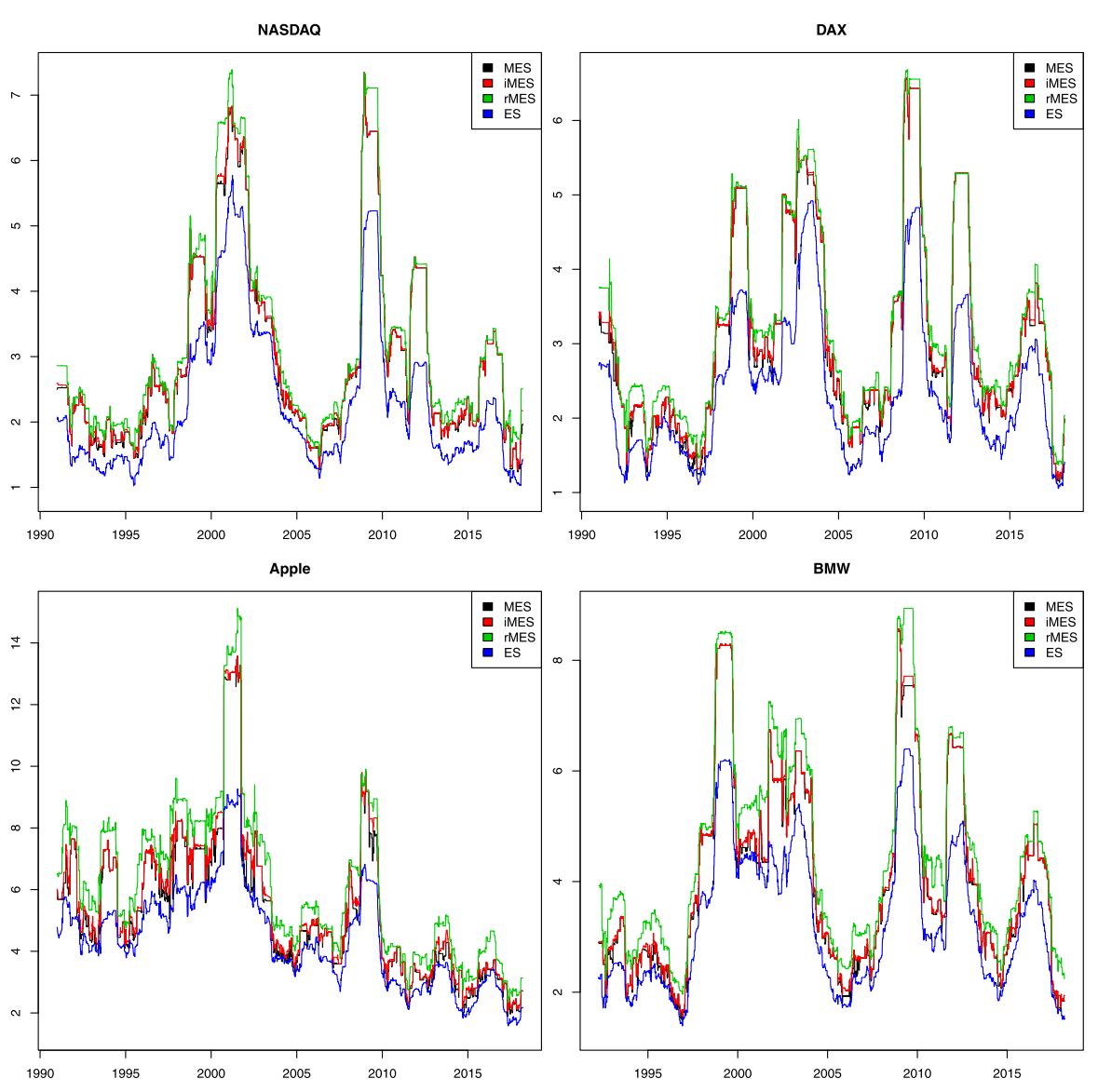

Fig. $1 \mathcal{Q}$-based risk measures estimated for data based on economic scenarios with $w=250$

then we subdivide the 125 days with high volatility in the current time window into two categories of (almost) equal size according to whether the S\&P 500 residuals are above or below their median during those 125 days. The same is then done for the 125 days with low volatility. This results in a split of the time window into four scenarios of (almost) equal size.

The sets $D_{1}, \ldots, D_{4}$ consist of the values of $X_{t}$ for $t=t_{0}-250, \ldots, t_{0}-1$ depending on which scenario the respective day has been assigned. We considered return data from the NASDAQ Composite Index, the DAX, Apple Inc. stock, and BMW stock. The data are freely available and were obtained from https://finance. yahoo.com. The considered time periods are 1991-2018. We do not consider data from before 1990 because there is no VIX data available. We chose the confidence level $p=0.9$ for simplicity. For each series of return data, we also computed the empirical $\mathrm{ES}_{p}$ using rolling windows of the same size. The results of the analysis are summarised in Fig. 1.

The risk measures $\mathrm{MES}_{p}$ and $\mathrm{iMES}_{p}$ generally yield similar values. This may be a hint that the empirical distributions under the different scenarios are often stochas- 
tically ordered. One can observe that during times of financial stress, the $\mathcal{Q}$-based expected shortfalls and $\mathrm{ES}_{p}$ deviate substantially, whereas they are closer during an economically stable period. For the indices (NASDAQ and DAX), iMES $_{p}$ and $\mathrm{rMES}_{p}$ are closer than for the stock returns (Apple and BMW). The risk measures $\mathrm{iMES}_{p}$ and $\mathrm{rMES}_{p}$ are close if the empirical distribution under one scenario strongly dominates the others in the sense that the quantile function under one scenario is much larger than under the others. Therefore, this phenomenon may be explained by the fact that the indices are more closely related to the quantities defining the economic scenarios (VIX and S\&P 500). It also explains why the ratio between $\mathrm{rMES}_{p}$ and $\mathrm{iMES}_{p}$ is in general larger during financial stress than in economically stable periods. The ratio between $\mathrm{MES}_{p}$ and $\mathrm{ES}_{p}$ qualitatively distinguishes the early 2000s recession from the 2008 financial crisis by being larger during the latter event, except for the Apple stock. Apple seems to have been more influenced by the dot-com crash in 2000 than the other stocks and indices.

\subsection{The Basel stress adjustment for expected shortfall}

In this section, we calculate the stress adjustment for expected shortfall in the Basel market risk evaluation as outlined in Sect. 1.1. Suppose that there are $n$ securities in a portfolio and let $P_{t}^{i}, i=1, \ldots, n, t \in \mathbb{N}$, denote the time- $t$ price of security $i$. Let $X_{t}^{i}=-\left(P_{t}^{i} / P_{t-1}^{i}-1\right)$ be its daily negative return. Construct a portfolio with price process $V_{t}=\sum_{i=1}^{n} \alpha_{i} P_{t}^{i}$, where $\alpha_{i}$ for $i=1, \ldots, n$ is the number of shares invested in security $i$, assumed fixed throughout the investment period. At time $t-1$, we need to calculate the empirical ES of the next day loss of this portfolio. The daily loss is

$$
V_{t-1}-V_{t}=\sum_{i=1}^{n} \alpha_{i}\left(P_{t-1}^{i}-P_{t}^{i}\right)=\sum_{i=1}^{n} X_{t}^{i} \alpha_{i} P_{t-1}^{i}
$$

At time $t-1$, the values $\alpha_{i}$ and $P_{t-1}^{i}$ are known, and the random risk factors are $\left(X_{t}^{1}, \ldots, X_{t}^{n}\right)$. To calculate the ES over the past 12 months of data, we need to evaluate the quantity, given the number $\alpha_{i} P_{t-1}^{i}$,

$$
\mathrm{ES}_{p}^{P}\left(V_{t-1}-V_{t}\right)=\mathrm{ES}_{p}^{P}\left(\sum_{i=1}^{n} X_{t}^{i} \alpha_{i} P_{t-1}^{i}\right)
$$

where $p=0.975$ as specified in [5]. For this purpose, the scenario $P$ is modelled such that the distribution of $\left(X_{t}^{1}, \ldots, X_{t}^{n}\right)$ is equal to its empirical version over the past 250 observations, i.e., over the period $[t-250, t-1]$.

ES should be calibrated to the most severe 12-month period of stress over a long observation horizon which has to span back to 2007, as specified in [5]. To mimic this adjustment for the period before the introduction of Basel III, it seems fair for everyday evaluation to look back 10 years and find the maximum ES over a 12-month period. For this purpose, we evaluate, while treating $\alpha_{i} P_{t-1}^{i}$ as a constant, the MaxES given by

$$
\operatorname{MES}_{p}^{\mathcal{Q}}\left(V_{t-1}-V_{t}\right)=\operatorname{MES}_{p}^{\mathcal{Q}}\left(\sum_{i=1}^{n} X_{t}^{i} \alpha_{i} P_{t-1}^{i}\right)=\max _{j=1, \ldots, N} \operatorname{ES}_{p}^{Q_{j}}\left(\sum_{i=1}^{n} X_{t}^{i} \alpha_{i} P_{t-1}^{i}\right)
$$




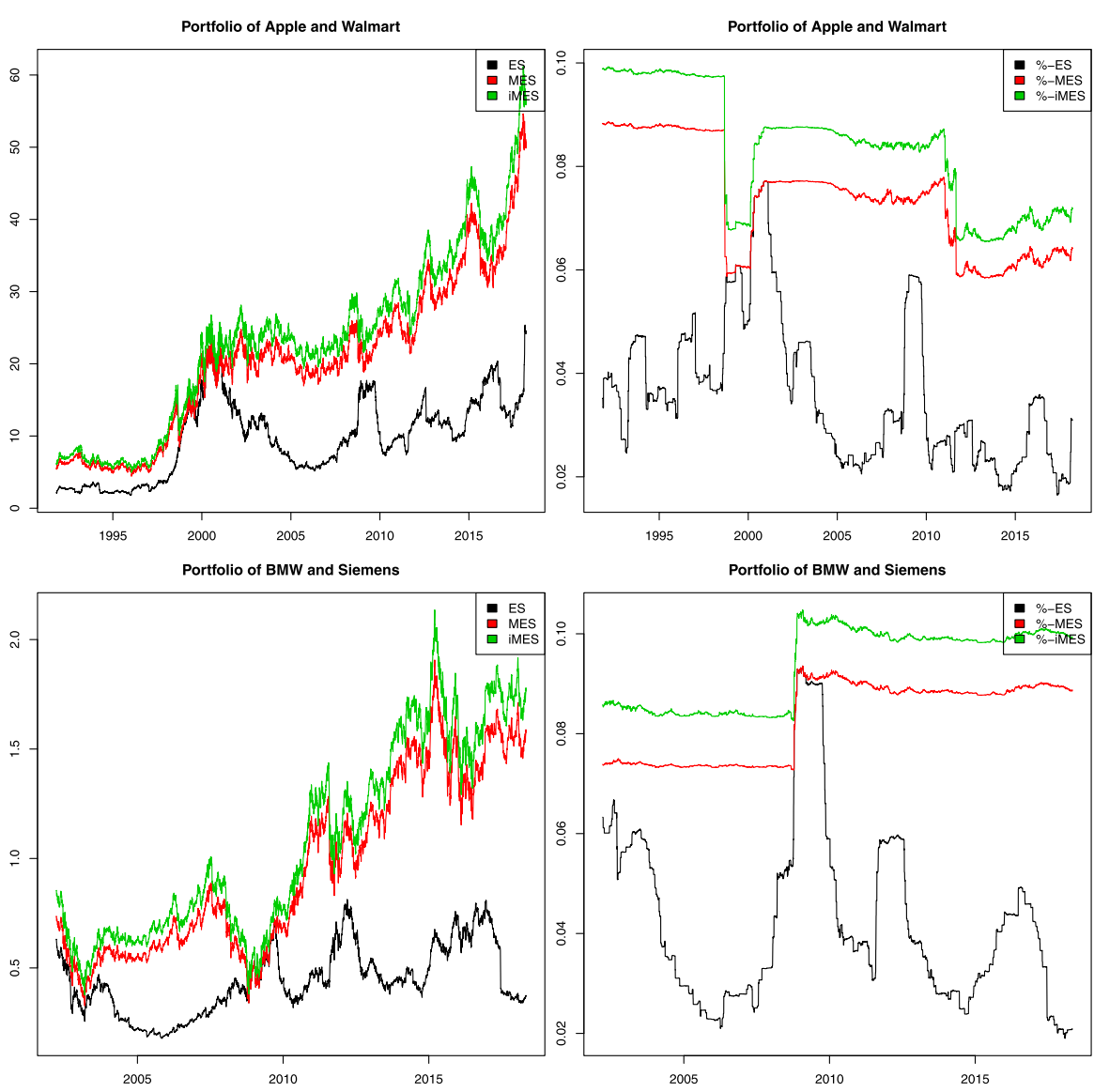

Fig. 2 The MES and ES of the US and German portfolios: (left) MES and ES of the portfolio; (right) the percentage of MES and ES in the value of the portfolio

where $N=2251, \mathcal{Q}=\left\{Q_{j}\right\}_{j=1, \ldots, N}$ and under $Q_{j},\left(X_{t}^{1}, \ldots, X_{t}^{n}\right)$ is distributed according to its empirical distribution over the time period $[t-j-249, t-j]$. Using the same scenarios, we also compute the integral Max-ES, that is, $\operatorname{iMES}_{p}^{\mathcal{Q}}\left(V_{t-1}-V_{t}\right)$. It is not sensible to compute the replicated Max-ES, $\operatorname{rMES}_{p}^{\mathcal{Q}}$ in this case since the number of scenarios is too large. We choose $\alpha_{1}, \ldots, \alpha_{n}$ such that each $\alpha_{i} P_{i}^{t}$ starts from $\$ 1$. We construct a US stocks portfolio (Apple and Walmart) and a German stocks portfolio (BMW and Siemens).

In Fig. 2, we report $\operatorname{ES}_{p}^{P}, \operatorname{MES}_{p}^{\mathcal{Q}}$ and $\operatorname{iMES}_{p}^{\mathcal{Q}}$ in the left panel, and the percentage of $\operatorname{ES}_{p}^{P}, \operatorname{MES}_{p}^{\mathcal{Q}}$ and $\operatorname{iMES}_{p}^{\mathcal{Q}}$, that is, $\operatorname{ES}_{p}^{P}\left(\left(V_{t-1}-V_{t}\right) / V_{t-1}\right)$ and so forth, in the right panel. The first row concerns the US and the second the German stocks portfolio.

The percentage $\operatorname{MES}_{p}^{\mathcal{Q}}$ and the percentage iMES ${ }_{p}^{\mathcal{Q}}$ are relatively stable (between $6 \%$ and $9 \%$, respectively $7 \%$ and $10 \%$ ), and the percentage $\operatorname{ES}_{p}^{P}$ is changing drastically (between $2 \%$ and $9 \%$ ), very much depending on the performance of the individual stocks over the past year. This suggests that $\operatorname{MES}_{p}^{\mathcal{Q}}$ and $\operatorname{iMES}_{p}^{\mathcal{Q}}$ have the 
advantage of being more robust since they are computed as worst cases over many past scenarios. The US portfolio has a quite high percentage $\operatorname{MES}_{p}^{Q}$ and $\operatorname{iMES}_{p}^{\mathcal{Q}}$ until 1998, and this is due to the effect of the Black Monday (October 19, 1987) that wears out after 10 years. The quotient of $\operatorname{MES}_{p}^{\mathcal{Q}}$ and $\mathrm{iMES}_{p}^{\mathcal{Q}}$ varies little over time, which is in contrast to the analysis in Sect. 5.1. Currently, we are lacking a clear theoretical explanation of this phenomenon.

If the regulatory capital for the market risk is calculated via $\operatorname{ES}_{p}^{P}$, then both portfolios exhibit serious under-capitalisation right before the 2007 financial crisis, and their $\operatorname{ES}_{p}^{P}$ values increased drastically when the financial crisis took place. On the other hand, if $\operatorname{MES}_{p}^{\mathcal{Q}}$ or iMES ${ }_{p}^{\mathcal{Q}}$ are used for regulatory capital calculation, then the requirement of capital for both portfolios only increased moderately during the financial crisis. From the data analysis, we do not see a clear advantage of $\operatorname{MES}_{p}^{\mathcal{Q}}$ over iMES ${ }_{p}^{\mathcal{Q}}$, or vice versa. However, $\operatorname{iMES}_{p}^{\mathcal{Q}}$ seems preferable from a theoretical perspective since it is comonotonic-additive.

\section{Concluding remarks}

In this paper, we propose a framework for scenario-based risk evaluation where different scenarios (probability measures or models) are incorporated into the procedure of risk calculation. Our framework allows flexible interpretation of the scenarios and is in particular motivated by the Basel calculation procedures for the expected shortfall, the Chicago Mercantile Exchange and the credit ratings, as explained in Sect. 1.1. Several theoretical contributions are made. We introduce new classes of risk measures including Max-ES, Max-VaR and their variants, and study their theoretical properties. Axiomatic characterisation of scenario-based comonotonic-additive and coherent classes of risk measures are obtained, and they are connected to the Basel formulas for market risk. Finally, we present data analyses to illustrate how scenariobased risk measures can be estimated, computed and interpreted.

Given the pivotal importance of model uncertainty and scenario analysis in modern risk management, scenario-based risk measures can be useful in many disciplines of risk assessment, not limited to financial risk management.

We remark that for various interpretations of the scenarios, the estimation procedures of a scenario-based risk measure may exhibit different properties, as illustrated in Sect. 5. This calls for future research in statistical theory for scenario-based risk functionals. A challenging open question is the characterisation of scenario-based coherent risk measures for general scenarios without assuming mutual singularity.

\section{Appendix A}

\section{A.1 Examples and counterexamples}

Example A.1 $\operatorname{MES}_{p}^{\mathcal{Q}}$ is not comonotonic-additive. To see this, fix $p \in(0,1)$ and take $Q_{1}, Q_{2} \in \mathcal{P}, A_{1}, A_{2} \in \mathcal{F}$ such that we have $A_{1} \subseteq A_{2}, Q_{1}\left[A_{1}\right]>Q_{2}\left[A_{1}\right]$ and also $Q_{1}\left[A_{2}\right]<Q_{2}\left[A_{2}\right]<1-p$. The existence of such $Q_{1}, Q_{2}, A_{1}, A_{2}$ can be shown 
by taking $\left(\Omega, \mathcal{F}, Q_{1}\right)$ and $\left(\Omega, \mathcal{F}, Q_{2}\right)$ as atomless probability spaces. Define the set $\mathcal{Q}=\left\{Q_{1}, Q_{2}\right\}, X=\mathbb{1}_{A_{1}}$ and $Y=\mathbb{1}_{A_{2}}$. It is clear that $X$ and $Y$ are comonotonic. Recall that for a Bernoulli random variable $Z$ under $Q$ with parameter $q$, we have $\operatorname{ES}_{p}^{Q}(Z)=q /(1-p)$. We have

$$
\begin{aligned}
\operatorname{ES}_{p}^{Q_{1}}(X+Y) & =\operatorname{ES}_{p}^{Q_{1}}(X)+\operatorname{ES}_{p}^{Q_{1}}(Y)=\frac{1}{1-p}\left(Q_{1}\left[A_{1}\right]+Q_{1}\left[A_{2}\right]\right) \\
& <\frac{1}{1-p}\left(Q_{1}\left[A_{1}\right]+Q_{2}\left[A_{2}\right]\right)=\max _{Q \in \mathcal{Q}} \operatorname{ES}_{p}^{Q}(X)+\max _{Q \in \mathcal{Q}} \operatorname{ES}_{p}^{Q}(Y)
\end{aligned}
$$

and similarly,

$$
\operatorname{ES}_{p}^{Q_{2}}(X+Y)<\max _{Q \in \mathcal{Q}} \operatorname{ES}_{p}^{Q}(X)+\max _{Q \in \mathcal{Q}} \operatorname{ES}_{p}^{Q}(Y)=\operatorname{MES}_{p}^{\mathcal{Q}}(X)+\operatorname{MES}_{p}^{\mathcal{Q}}(Y) .
$$

Then we have

$$
\operatorname{MES}_{p}^{\mathcal{Q}}(X+Y)=\max \left\{\operatorname{ES}_{p}^{Q_{1}}(X+Y), \operatorname{ES}_{p}^{Q_{2}}(X+Y)\right\}<\operatorname{MES}_{p}^{\mathcal{Q}}(X)+\operatorname{MES}_{p}^{\mathcal{Q}}(Y) .
$$

Thus $\operatorname{MES}_{p}^{\mathcal{Q}}$ is not comonotonic-additive.

Example A.2 We have $\operatorname{MES}_{p}^{\mathcal{Q}}(X)<\operatorname{ES}_{p}^{\mathbb{P}}(X)$ for $\mathcal{Q}$ in Example 2.1. Consider a set $\Omega=\left\{\omega_{1}, \ldots, \omega_{8}\right\}$ with eight pairwise distinct elements, and let $\mathbb{P}$ be the uniform probability measure on $\Omega$. Write $\Omega_{1}=\left\{\omega_{1}, \ldots, \omega_{4}\right\}$ and $\Theta=\mathbb{1}_{\Omega_{1}}$. Let $Q_{1}[\cdot]=\mathbb{P}[\cdot \mid \Theta=1], Q_{2}[\cdot]=\mathbb{P}[\cdot \mid \Theta=0]$ and $X=\mathbb{1}_{\Omega_{1}}+2 \mathbb{1}_{\left\{\omega_{8}\right\}}$. It is easy to see

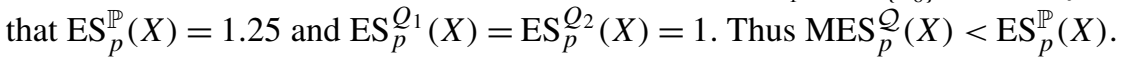

\section{A.2 Extension of Proposition 4.6}

We show here that parts (i), (ii) and (iv) of Proposition 4.6 also hold if $\mathcal{Q}$ is infinite.

Concerning (i), $\operatorname{ES}_{p}^{Q}$ is coherent for $Q \in \mathcal{Q}$. Since $\operatorname{MES}_{p}^{Q}$ can be written as a supremum of coherent risk measures and taking a supremum preserves all properties of coherent risk measures, $\operatorname{MES}_{p}^{\mathcal{Q}}$ is also coherent. An example showing that $\operatorname{MES}_{p}^{\mathcal{Q}}$ is not comonotonic-additive is given in Example A.1. Concerning (ii), $\operatorname{VaR}_{p}^{Q}$ is monetary for $Q \in \mathcal{Q}$, and hence $\operatorname{MVaR}_{p}^{\mathcal{Q}}$ as a supremum of monetary risk measures is monetary as well. It remains to show that $\operatorname{MVaR}_{p}^{\mathcal{Q}}$ is a comonotonic-additive risk measure. Using Denneberg's lemma [13, Proposition 4.5], for comonotonic random variables $X$ and $Y$, there exist increasing continuous functions $f$ and $g$ such that $X=f(X+Y)$ and $Y=g(X+Y)$. Therefore, for any $Q \in \mathcal{Q}$, we have

$$
\begin{aligned}
\operatorname{MVaR}_{p}^{\mathcal{Q}}(X) & =\sup _{Q \in \mathcal{Q}} \operatorname{VaR}_{p}^{Q}(f(X+Y)) \\
& =\sup _{Q \in \mathcal{Q}} f\left(\operatorname{VaR}_{p}^{Q}(X+Y)\right)=f\left(\sup _{Q \in \mathcal{Q}} \operatorname{VaR}_{p}^{Q}(X+Y)\right),
\end{aligned}
$$


and similarly,

$$
\operatorname{MVaR}_{p}^{\mathcal{Q}}(Y)=g\left(\sup _{Q \in \mathcal{Q}} \operatorname{VaR}_{p}^{Q}(X+Y)\right)
$$

Note that $f(z)+g(z)=z$ for $z$ in the range of $X+Y$, and by continuity, $f$ and $g$ also satisfy $f(z)+g(z)=z$ for $z=\sup _{Q \in \mathcal{Q}} \operatorname{VaR}_{p}^{Q}(X+Y)$. Hence we have

$$
\begin{aligned}
\operatorname{MVaR}_{p}^{\mathcal{Q}}(X+Y) & =\sup _{Q \in \mathcal{Q}} \operatorname{VaR}_{p}^{Q}(X+Y) \\
& =f\left(\sup _{Q \in \mathcal{Q}} \operatorname{VaR}_{p}^{Q}(X+Y)\right)+g\left(\sup _{Q \in \mathcal{Q}} \operatorname{VaR}_{p}^{Q}(X+Y)\right) \\
& =\operatorname{MVaR}_{p}^{\mathcal{Q}}(X)+\operatorname{MVaR}_{p}^{\mathcal{Q}}(Y) .
\end{aligned}
$$

The statement that $\operatorname{MVaR}_{p}^{\mathcal{Q}}$ is not necessarily coherent comes from the well-known fact that $\operatorname{VaR}_{p}^{Q}$ is not coherent for any $Q \in \mathcal{P}$ such that $(\Omega, \mathcal{F}, Q)$ is atomless. Concerning (iv), it suffices to note that $\mathrm{iMES}_{p}^{\mathcal{Q}}$ is a mixture of comonotonic-additive risk measures and hence is comonotonic-additive as well.

Acknowledgements The authors thank the Editor, an Associate Editor, two anonymous referees, Rama Cont, Paul Embrechts, Damir Filipović, Steven Kou, Fabio Maccheroni, Marco Maggis, Ilya Molchanov and Andreas Tsanakas for helpful discussions on an earlier version of the present paper. Wang acknowledges financial support from the Natural Sciences and Engineering Research Council of Canada (RGPIN2018-03823/RGPAS-2018-522590).

Funding Note Open Access funding provided by Universität Bern.

Open Access This article is licensed under a Creative Commons Attribution 4.0 International License, which permits use, sharing, adaptation, distribution and reproduction in any medium or format, as long as you give appropriate credit to the original author(s) and the source, provide a link to the Creative Commons licence, and indicate if changes were made. The images or other third party material in this article are included in the article's Creative Commons licence, unless indicated otherwise in a credit line to the material. If material is not included in the article's Creative Commons licence and your intended use is not permitted by statutory regulation or exceeds the permitted use, you will need to obtain permission directly from the copyright holder. To view a copy of this licence, visit http://creativecommons.org/licenses/by/ $4.0 \%$.

\section{References}

1. Acharya, V., Engle, R., Richardson, M.: Capital shortfall: a new approach to ranking and regulating systemic risks. Am. Econ. Rev. 102, 59-64 (2012)

2. Adrian, T., Brunnermeier, M.K.: CoVaR. Am. Econ. Rev. 106, 1705-1741 (2016)

3. Anscombe, F.J., Aumann, R.J.: A definition of subjective probability. Ann. Math. Stat. 34, 199-205 (1963)

4. Artzner, P., Delbaen, F., Eber, J.-M., Heath, D.: Coherent measures of risk. Math. Finance 9, 203-228 (1999)

5. BCBS (Basel Committee on Banking Supervision): Standards. Minimum Capital Requirements for Market Risk. January 2016. Available online at https://www.bis.org/bcbs/publ/d352.htm

6. BCBS (Basel Committee on Banking Supervision): Frequently Asked Questions on Market Risk Capital Requirements. January 2017. Available online at https://www.bis.org/bcbs/publ/d437.htm 
7. Cambou, M., Filipović, D.: Model uncertainty and scenario aggregation. Math. Finance 27, 534-567 (2017)

8. Cerreia-Vioglio, S., Maccheroni, F., Marinacci, M., Montrucchio, L.: Ambiguity and robust statistics. J. Econ. Theory 148, 974-1049 (2013)

9. CME (Chicago Mercantile Exchange): CME SPAN: Standard Portfolio Analysis of Risk, (2010). Available online at https://www.cmegroup.com/clearing/span-methodology.html

10. Cherny, A.S., Madan, D.: New measures for performance evaluation. Rev. Financ. Stud. 22, 2571-2606 (2009)

11. Delbaen, F.: Coherent risk measures on general probability spaces. In: Sandmann, K., Schönbucher, P.J. (eds.) Advances in Finance and Stochastics. Essays in Honour of Dieter Sondermann, pp. 1-37. Springer, Berlin (2002)

12. Delbaen, F.: Monetary Utility Functions. Osaka University Press, Osaka (2012)

13. Denneberg, D.: Non-additive Measure and Integral. Springer, Berlin (1994)

14. Dhaene, J., Kukush, A., Linders, D., Tang, Q.: Remarks on quantiles and distortion risk measures. Eur. Actuar. J. 2, 319-328 (2012)

15. Du, Z., Escanciano, J.C.: Backtesting expected shortfall: accounting for tail risk. Manag. Sci. 63, 940-958 (2017)

16. Duffie, D., Scheicher, M., Vuillemey, G.: Central clearing and collateral demand. J. Financ. Econ. 116, 237-256 (2015)

17. Embrechts, P., Liu, H., Wang, R.: Quantile-based risk sharing. Oper. Res. 66, 936-949 (2018)

18. Embrechts, P., Puccetti, G., Rüschendorf, L., Wang, R., Beleraj, A.: An academic response to Basel 3.5. Risks 2, 25-48 (2014)

19. Embrechts, P., Wang, B., Wang, R.: Aggregation-robustness and model uncertainty of regulatory risk measures. Finance Stoch. 19, 763-790 (2015)

20. Fissler, T., Ziegel, J.F.: Higher order elicitability and Osband's principle. Ann. Stat. 44, 1680-1707 (2016)

21. Föllmer, H., Schied, A.: Convex measures of risk and trading constraints. Finance Stoch. 6, 429-447 (2002)

22. Föllmer, H., Schied, A.: Stochastic Finance. An Introduction in Discrete Time, 4th edn. de Gruyter, Berlin (2016)

23. Gilboa, I., Schmeidler, D.: Maxmin expected utility with non-unique prior. J. Math. Econ. 18, 141-153 (1989)

24. Guo, N., Kou, S., Wang, B., Wang, R.: Self-consistency, subjective pricing, and a theory of credit rating. Preprint (2019). Available online at https://ssrn.com/abstract=3504065

25. Hansen, L.P., Marinacci, M.: Ambiguity aversion and model misspecification: an economic perspective. Stat. Sci. 31, 511-515 (2016)

26. IAIS: Consultation Document December 2014. Risk-based Global Insurance Capital Standard. International Association of Insurance Supervisors (2014). Available online at https://www.iaisweb.org/ file/41483/risk-based-global-insurance-capital-standard-consultation-document

27. Jouini, E., Schachermayer, W., Touzi, N.: Law invariant risk measures have the Fatou property. Adv. Math. Econ. 9, 49-71 (2006)

28. Kou, S., Peng, X.: On the measurement of economic tail risk. Oper. Res. 64, 1056-1072 (2016)

29. Kou, S., Peng, X., Heyde, C.C.: External risk measures and Basel Accords. Math. Oper. Res. 38, 393-417 (2013)

30. Krätschmer, V., Schied, A., Zähle, H.: Domains of weak continuity of statistical functionals with a view toward robust statistics. J. Multivar. Anal. 158, 1-19 (2017)

31. Kusuoka, S.: On law invariant coherent risk measures. Adv. Math. Econ. 3, 83-95 (2001)

32. Li, L., Xing, H.: Capital allocation under the Fundamental Review of Trading Book. Risk Magazine, 06 Jun 2019 (2019)

33. McNeil, A.J., Frey, R., Embrechts, P.: Quantitative Risk Management: Concepts, Techniques and Tools, Princeton University Press, Princeton (2015). Revised edn.

34. McNeil, A.J., Nešlehová, J.: Multivariate Archimedean copulas, $d$-monotone functions and $l_{1}$-norm symmetric distributions. Ann. Stat. 37, 3059-3097 (2009)

35. Müller, A., Stoyan, D.: Comparison Methods for Stochastic Models and Risks. Wiley, England (2002)

36. OCC (Office of the Comptroller of the Currency): Supervisory Guidance on Model Risk Management. OCC 2011-12, Board of Governors of the Federal Reserve System (2011). Available online at https:// www.occ.treas.gov/news-issuances/bulletins/2011/bulletin-2011-12a.pdf

37. Righi, M.B.: A theory for combinations of risk measures. Preprint (2020). Available online at https:// arxiv.org/abs/1807.01977 
38. Rockafellar, R.T., Uryasev, S., Zabarankin, M.: Generalized deviations in risk analysis. Finance Stoch. 10, 51-74 (2006)

39. Rüschendorf, L.: Mathematical Risk Analysis. Dependence, Risk Bounds, Optimal Allocations and Portfolios. Springer, Heidelberg (2013)

40. Schmeidler, D.: Integral representation without additivity. Proc. Am. Math. Soc. 97, 255-261 (1986)

41. Shen, J., Shen, Y., Wang, B., Wang, R.: Distributional compatibility for change of measures. Finance Stoch. 23, 761-794 (2019)

42. Wang, R., Zitikis, R.: An axiomatic foundation for the Expected Shortfall. Manag. Sci. 67, 1413-1429 (2020)

43. Yaari, M.E.: The dual theory of choice under risk. Econometrica 55, 95-115 (1987)

44. Zhu, S., Fukushima, M.: Worst-case conditional Value-at-Risk with applications to robust portfolio management. Oper. Res. 57, 1155-1168 (2009)

45. Zymler, S., Kuhn, D., Rustem, B.: Worst-case Value at Risk of nonlinear portfolios. Manag. Sci. 59, 172-188 (2012)

Publisher's Note Springer Nature remains neutral with regard to jurisdictional claims in published maps and institutional affiliations. 